\title{
The Evolution of Tumour Composition During Fractionated Radiotherapy: Implications for Outcome
}

\author{
Thomas D. Lewin $^{1}$ (D) Philip K. Maini ${ }^{1}$ • Eduardo G. Moros ${ }^{2}$ • \\ Heiko Enderling ${ }^{3}$. Helen M. Byrne ${ }^{1}$
}

Received: 4 August 2017 / Accepted: 8 January 2018 / Published online: 27 February 2018 (C) The Author(s) 2018. This article is an open access publication

\begin{abstract}
Current protocols for delivering radiotherapy are based primarily on tumour stage and nodal and metastases status, even though it is well known that tumours and their microenvironments are highly heterogeneous. It is well established that the local oxygen tension plays an important role in radiation-induced cell death, with hypoxic tumour regions responding poorly to irradiation. Therefore, to improve radiation response, it is important to understand more fully the spatiotemporal distribution of oxygen within a growing tumour before and during fractionated radiation. To this end, we have extended a spatially resolved mathematical model of tumour growth, first proposed by Greenspan (Stud Appl Math 51:317-340, 1972), to investigate the effects of oxygen heterogeneity on radiation-induced cell death. In more detail, cell death due to radiation at each location in the tumour, as determined by the well-known linear-quadratic model, is assumed also to depend on the local oxygen concentration. The oxygen concentration is governed by a reaction-diffusion equation that is coupled to an integro-differential equation that determines the size of the assumed spherically symmetric tumour. We combine numerical and analytical techniques to investigate radiation response of tumours with different intratumoral oxygen distribution profiles. Model simulations reveal a rapid transient increase in hypoxia upon regrowth of the tumour spheroid post-irradiation. We investigate the response to different radiation fractionation schedules and identify a tumour-specific relationship
\end{abstract}

Thomas D. Lewin

lewint@maths.ox.ac.uk

1 Wolfson Centre for Mathematical Biology, Mathematical Institute, University of Oxford, Oxford, UK

2 Radiation Oncology, H. Lee Moffitt Cancer Center \& Research Institute, Tampa, FL, USA

3 Integrated Mathematical Oncology, H. Lee Moffitt Cancer Center \& Research Institute, Tampa, FL, USA 
between inter-fraction time and dose per fraction to achieve cure. The rich dynamics exhibited by the model suggest that spatial heterogeneity may be important for predicting tumour response to radiotherapy for clinical applications.

Keywords Fractionated radiotherapy · Avascular tumours · Moving boundaries · Hypoxia

\section{Introduction}

Cancer remains one of the main causes of mortality worldwide, with an estimated 8.2 million deaths in 2012 (Torre et al. 2015). Of the millions of people diagnosed with some form of cancer each year, about half will receive radiotherapy as part of their treatment (Fowler 2006). Typically, radiation is delivered to the tumour as a series of small doses, or fractions, administered over a period of several days or weeks in order to limit the toxic side effects to healthy cell populations. The conventional fractionation schedule for most tumours, where a dose of approximately 2 Gy (Gray) is delivered once a day Monday to Friday up to a total of 50-70 Gy, has remained the standard of care for many years (Ahmed et al. 2014; Marcu 2010). While altered schemes, such as hyperfractionation, accelerated fractionation and hypofractionation, have been proposed for certain indications, the selection of an optimal fractionation protocol for a particular tumour would clearly benefit from a more individualised approach. In particular, beyond tumour location and stage, patient-specific factors that may be important in determining response to a particular treatment are currently not considered. It is in this regard that mathematical modelling has the potential to play an important role, identifying key factors that determine treatment outcomes and ultimately identifying patient-specific treatment protocols.

Tumours differ in their responses to irradiation, with radiosensitivity being understood to be an intrinsic property of the cell population that could be estimated from molecular analysis of biopsy samples (Eschrich et al. 2009). Other factors that affect a tissue's response to radiotherapy include repopulation between radiation doses, tissue re-oxygenation and redistribution of cells within the cell cycle (Thrall 1997). For rapidly proliferating tumours in particular, repopulation between fractions of radiation is important. For tumours containing larger regions of hypoxia, re-oxygenation may play a pivotal role. A more detailed description of the underlying radiobiology can be found in Joiner and van der Kogel (2009).

Due to the difficulties of studying tumours in vivo, such effects are often investigated experimentally using in vitro multicellular tumour spheroids. Tumour spheroids represent a controlled environment of intermediate complexity between $2 \mathrm{D}$ culture media and in vivo models in which important insight can be gained about the tumour micro-environment and the effect of therapies on a growing tumour (Carlson et al. 2006; Hirschhaeuser et al. 2010; Mueller-Klieser 1987; Sutherland et al. 1981). However, tumour spheroid growth is avascular with a typical diameter of less than $5 \mathrm{~mm}$, so while useful for calibrating mathematical models, parameters derived from tumour spheroids may need adjustment before they can be used to simulate in vivo tumours. 
The most widely adopted mathematical model of radiotherapy response is the linearquadratic (LQ) model, which is observed to correspond well with survival curves obtained experimentally from clonogenic assays (Joiner and van der Kogel 2009). We note that although the LQ model is empirical, mechanistic models have been proposed to explain it (Sachs et al. 1997; Nilsson et al. 1990; Joiner and van der Kogel 2009). In the LQ model, the survival fraction, sf, of tumour cells after a dose, $d \mathrm{~Gy}$, of radiation is given by

$$
\operatorname{sf}(d)=e^{-\alpha d-\beta d^{2}}
$$

where $\alpha\left(\mathrm{Gy}^{-1}\right)$ and $\beta\left(\mathrm{Gy}^{-2}\right)$ are intrinsic radiosensitivity parameters (Fowler 2006; Sachs et al. 1997; Withers 1999; O'Rourke et al. 2009). Values for $\alpha$ and $\beta$ may differ significantly between tumours and patients. The $\alpha / \beta$ (Gy) ratio is often used to characterise the sensitivity of a particular tissue type to fractionation. Values of $\alpha / \beta$ can fall as low as $1 \mathrm{~Gy}$ for late-responding tissues such as prostate cancer, and reach as high as 20 Gy for early-responding, rapidly proliferating tissues such as head and neck cancer (Withers 1999). The LQ model is frequently incorporated into more detailed tumour models as an instantaneous effect (McAneney and O'Rourke 2007; Prokopiou et al. 2015).

Many different mathematical models and methodologies have been used to study tumour growth. Among the simplest of these are ordinary differential equation (ODE) models that aim to qualitatively capture observed growth dynamics. Tumours of small volume typically grow exponentially, and their growth rates decelerate as microenvironmental factors such as limited space and nutrient availability become important, which leads ultimately to sigmoidal patterns of growth (Sachs et al. 2001; McAneney and O'Rourke 2007). Popular ODE models include the logistic and Gompertz growth models where the volume-saturation limit is represented by the carrying capacity, $K$.

In many existing models of (avascular) tumour growth, the tumour is assumed to be spatially homogeneous. Such phenomenological models contain few parameters and do not account for complex underlying biological interactions. While such models may provide limited biological insight, the ease with which model parameters may be estimated from limited clinical data makes them attractive for making predictions about radiotherapy response and identifying personalised fractionation protocols in the clinic (Prokopiou et al. 2015).

A variety of more complex, spatially resolved, models have been proposed in order to provide more mechanistic insight, often reflecting the heterogeneous nature of growing tumours. In more detail, as a tumour grows, it will typically develop hypoxia and necrosis in regions where oxygen or nutrient supply is inadequate. Multicellular, avascular tumour spheroids are used as in vitro models to study, in a controlled environment, effects observed in vivo (Carlson et al. 2006; Hirschhaeuser et al. 2010; Mueller-Klieser 1987; Sutherland et al. 1981; Folkman and Hochberg 1973). In a similar manner, it is natural to develop mathematical models for avascular tumour spheroids before considering the more complex case of vascular tumour growth.

One of the simplest spatially resolved models of avascular tumour growth was proposed in Greenspan (1972). In the Greenspan model, under the assumption of spherical symmetry, the outer tumour radius evolves in response to a single diffusible, growth-rate-limiting nutrient, commonly taken to be oxygen. Internal free boundaries, 
decomposing the tumour spheroid into a central necrotic core, an outer proliferating rim and an intermediate hypoxic annulus, are identified as contours of the oxygen profile across the tumour.

Under certain simplifying assumptions, Greenspan's model can be reduced to a coupled system of ODEs and algebraic equations. Most other spatially resolved continuum models are formulated as systems of partial differential equations (PDEs). Such approaches include frameworks from multiphase modelling and morphoelasticity. Multiphase models consider a mixture of two or more continuum constituents, or phases, which collectively comprise the tumour environment. Systems of PDEs are obtained by applying mass and momentum balances to each phase and making suitable constitutive assumptions about their mechanical properties and interactions (Breward et al. 2003; Byrne et al. 2003; Preziosi and Tosin 2009). Morphoelastic models of tumour growth provide a theoretical framework within which to study biological tissues for which growth and elasticity are inter-related (Araujo and McElwain 2004).

While incorporating more biological detail, these more sophisticated approaches typically involve more parameters that may be difficult to estimate in practice. In this paper, we present a simple spatially resolved model for tumour spheroid growth and response to radiotherapy in order to investigate the effects of spatial heterogeneity. In Sect. 2, we develop the model, extending Greenspan's original spatial model for tumour spheroid growth (Greenspan 1972) to include radiation effects. In Sect. 3.1, we numerically solve the model equations and discuss key features of the resulting dynamics. We present further numerical simulations and model analysis in order to understand the tumour dynamics exhibited by the model. In Sect. 3.2, we study tumour regrowth following a single dose of radiation, and in Sect. 3.3, we consider the longterm behaviour of a tumour for different fractionation schedules.

\section{Model Development}

In this section, we introduce a spatial model of avascular tumour growth originally presented in Greenspan (1972). Although many spatially resolved models that account for tumour growth and hypoxia have been proposed, a significant portion of these are based upon Greenspan's original work, and it remains a simple, yet useful starting point for investigating the effect of hypoxia on tumour responses to radiotherapy. Accordingly, in this paper we extend Greenspan's model to account for the effects of radiation and arrive at a new model for tumour response to radiotherapy.

\subsection{Summary of Greenspan's Original Model}

We consider the growth of a radially symmetric, avascular tumour spheroid in response to a single, growth-rate-limiting, diffusible nutrient, here oxygen. Following Greenspan 1972, we assume that growth inhibition is caused by nutrient deficiency rather than a tumour-derived inhibitory factor. We denote the outer tumour radius by $R(t)$ and the oxygen concentration at a distance $0 \leq r \leq R(t)$ from the tumour centre by $c(r, t)$. We suppose that the oxygen concentration is maintained at a constant level, $c_{\infty}$, on $r=R(t)$. Oxygen diffuses on a much shorter timescale than tumour growth, 


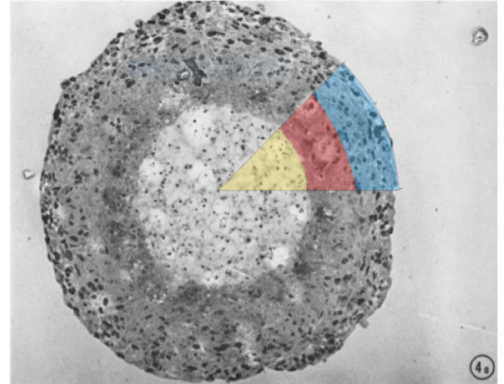

(a)

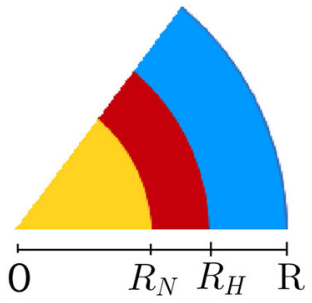

(b)

Fig. 1 Diagrams highlighting the distinct regions within a tumour spheroid and how they are influenced by the oxygen profile. A central necrotic core $\left(0<r<R_{N}\right)$ is surrounded by a hypoxic annulus $\left(R_{N}<r<\right.$ $\left.R_{H}\right)$ and an outer proliferating $\operatorname{rim}\left(R_{H}<r<R\right)$. The moving boundaries $r=R_{N}(t), R_{H}(t)$ and $R(t)$ delineate these regions. a Image of an in vitro tumour spheroid highlighting the distinct micro-environments (adapted from Folkman and Hochberg 1973), b schematic of the corresponding model tumour spheroid geometry

taking $\sim 10$ s to diffuse $100 \mu \mathrm{m}$ by comparison with a tumour growth period measured in days (Greenspan 1972). As such we assume that the oxygen concentration is in a quasi-steady state. Internal free boundaries at $r=R_{H}(t)$ and $r=R_{N}(t)$ are defined implicitly and denote contours on which the oxygen concentration attains the threshold values $c_{H}$ and $c_{N}$, respectively. These interfaces decompose a well-developed tumour into a central necrotic core $\left(0<r<R_{N}\right)$ where $c \leq c_{N}$, and an outer, oxygen-rich region $\left(R_{H}<r<R\right)$ in which $c_{H}<c$, these regions being separated by a hypoxic annulus $\left(R_{N}<r<R_{H}\right)$ in which $c_{N}<c<c_{H}$ (see Fig. 1 for a schematic).

Greenspan's original model (Greenspan 1972) describes how the dependent variables $c(r, t), R(t), R_{H}(t)$ and $R_{N}(t)$ evolve over time and can be written in dimensionless form as follows:

$$
\begin{aligned}
& 0=\underbrace{\frac{1}{r^{2}} \frac{\partial}{\partial r}\left(r^{2} \frac{\partial c}{\partial r}\right)}_{\text {diffusion term }}-\underbrace{\Gamma H\left(c-c_{N}\right)}_{\text {oxygen consumption }}, \\
& \underbrace{\frac{1}{4 \pi} \frac{\mathrm{d}}{\mathrm{d} t}\left(\frac{4 \pi R^{3}}{3}\right)}_{\text {rate of change of tumour volume }}=\int_{0}^{R}[\underbrace{c H\left(c-c_{H}\right)}_{\text {cell proliferation term }}-\underbrace{\lambda_{A}-\lambda_{N} H\left(c_{N}-c\right)}_{\text {cell death term }}] r^{2} \mathrm{~d} r, \\
& R_{H}=0 \text { if } c>c_{H} \forall r \text { and otherwise } c\left(R_{H}, t\right)=c_{H} \text {, } \\
& R_{N}=0 \text { if } c>c_{N} \forall r \text { and otherwise } c\left(R_{N}, t\right)=c_{N} \text {, } \\
& \frac{\partial c}{\partial r}=0 \text { at } r=0 \text {, } \\
& c=c_{\infty} \text { at } r=R, \\
& R(0)=R_{0} \text {, }
\end{aligned}
$$


where $H($.$) is the Heaviside function [ H(x)=1$ for $x>0$, and $H(x)=0$ otherwise], and $\Gamma, \lambda_{A}, \lambda_{N}, c_{\infty}, c_{H}$ and $c_{N}$ are positive constants, with $\Gamma, \lambda_{A}$ and $\lambda_{N}$ corresponding to rates of oxygen consumption, apoptosis and necrosis, respectively. Equations (2)-(8) may be reduced to a system involving a single ODE for $R(t)$ and algebraic equations for $R_{H}(t)$ and $R_{N}(t)$. Analysis of the resulting equations and details of the nondimensionalisation may be found in Greenspan (1972) and Byrne (2012), and for further explanation of the underlying model assumptions, see Appendix A.1. Figure 2 shows how the size and composition of the tumour spheroid evolve for a typical simulation of the Greenspan model using the dimensionless parameter values in Table 1. A table of dimensional parameter values is given in Appendix A.1. Where possible, parameter estimates are taken from the literature; however, we note that some values pertain to estimates used in other modelling scenarios. In this paper, specific parameter combinations serve only to highlight the dynamics of the model and, in particular, the resulting model analysis is independent of the choice of parameter values. The influence of some of the parameters on the growth dynamics and steady state tumour composition is explored in Appendix A.2.

\subsection{Incorporating Radiotherapy into the Greenspan Model}

We adapt Greenspan's model to account for radiotherapy by assuming that when a dose of radiotherapy is applied, it causes an instantaneous dose-dependent death of viable cancer cells and, thus, a change in the size and composition of the tumour. The efficacy of the radiation and subsequent cell death depends on the local oxygen concentration. As such, we determine the radiation-induced cell death in each tumour region separately. We denote by $R^{ \pm}, R_{H}^{ \pm}$and $R_{N}^{ \pm}$the radii immediately before (-) and after $(+)$ radiotherapy in the proliferating, hypoxic and necrotic tumour compartments.

\subsubsection{Radiation-Induced Cell Death and the Linear-Quadratic Model}

We use the linear-quadratic model (Fowler 2006; Joiner and van der Kogel 2009; Enderling et al. 2010) to account for cell kill due to radiotherapy in the well-oxygenated outer rim, $R_{H}<r<R$, so that the volume survival fraction, $\operatorname{sf}(d)$, immediately after a dose $d$ of radiation is given by

$$
\begin{aligned}
\mathrm{sf}_{\text {normoxic }} & =\frac{\text { [volume of normoxic region after radiotherapy }]}{\text { [volume of normoxic region before radiotherapy }]} \\
& =\exp \left(-\alpha d-\beta d^{2}\right)
\end{aligned}
$$

for radiosensitivity parameters $\alpha$ and $\beta$. Values of $\alpha / \beta$ vary markedly, with typical values falling in the range of 3-10 Gy. However, extremal values of 1 and 20 Gy have been reported for late-responding tissues such as prostate cancer, and early-responding, rapidly proliferating tissues such as head and neck cancer, respectively (Withers 1999). Typical radiosensitivity parameters for rapidly proliferating, early-responding tumours are shown in Table 2. 

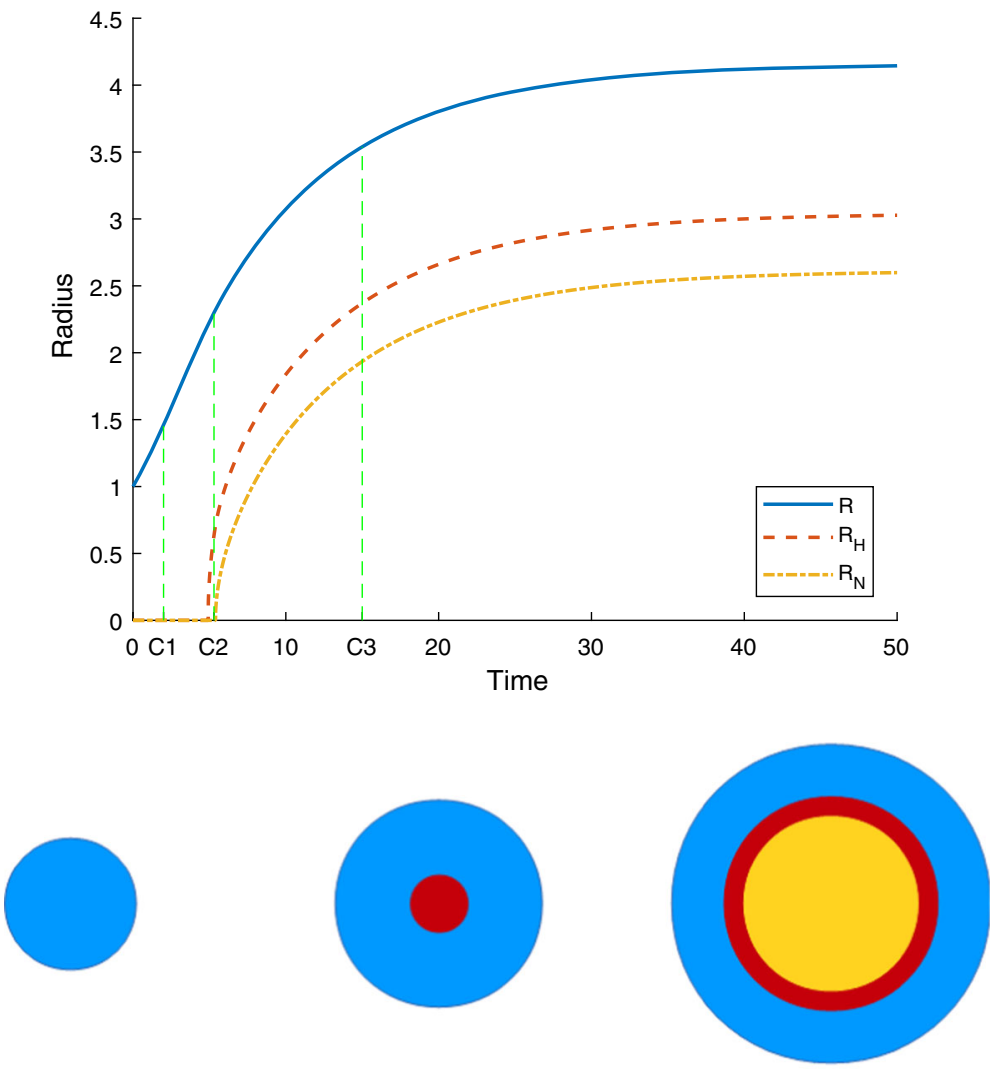

C1

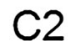

C3

Fig. 2 Results from a typical simulation of Eqs. (2)-(8) showing how the size and composition of the tumour spheroid change over time (parameter values in Table 1). Key: blue solid line-proliferating cells, red dashed line-hypoxic region, yellow dot/dashed line-necrotic core. The schematics represent the tumour compositions $\mathrm{C} 1-\mathrm{C} 3$ as the spheroid progresses through different stages of growth

Table 1 Dimensionless parameter values for tumour spheroid growth model

\begin{tabular}{llll}
\hline Parameter & Symbol & Value & Source \\
\hline Oxygen consumption rate & $\Gamma$ & 1.1051 & Grimes et al. (2014) \\
Oxygen concentration at tumour boundary & $c_{\infty}$ & 1 & Grimes et al. (2014) \\
Hypoxic oxygen threshold & $c_{H}$ & 0.1 & Grimes et al. (2014) \\
Anoxic oxygen threshold & $c_{N}$ & 0.008 & Grimes et al. (2014) \\
Apoptosis constant & $\lambda_{A}$ & 0.32 & Frieboes et al. (2007) \\
Necrosis constant & $\lambda_{N}$ & 0.0061 & Schaller and Meyer-Hermann (2006) \\
\hline
\end{tabular}

When exposed to ionising radiation, the potent oxygen free radicals that form in well-oxygenated regions increase the amount of DNA damage by up to a factor of 3 when compared with hypoxic tumour regions (Alper and Howard-Flanders 1956). 
Table 2 Typical radiosensitivity parameters

\begin{tabular}{lll}
\hline Parameter & Value & Source \\
\hline$\alpha\left(\mathrm{Gy}^{-1}\right)$ & 0.35 & Fowler (2006), Muriel (2006), Sachs et al. (2001) \\
$\alpha / \beta(\mathrm{Gy})$ & 10 & Withers (1999) \\
OER (dimensionless) & 3 & Carlson et al. (2006) \\
\hline
\end{tabular}

Following Carlson et al. (2006), we incorporate the oxygen enhancement ratio, OER, to account for this effect. Radiosensitivity parameters are typically quoted for normoxic conditions. As such we take the common approach and use the OER as a constant factor (taken to be equal to 3 in the presented simulations) that reduces the intrinsic radiosensitivity parameters of tumour cells, $\alpha$ and $\beta$, in hypoxic regions. This creates a discontinuity in the response to radiotherapy at $r=R_{H}$. The volume survival fraction immediately after a dose of radiation is delivered to a population of hypoxic cells is given by

$$
\begin{aligned}
\mathrm{sf}_{\text {hypoxic }} & =\frac{[\text { volume of hypoxic region after radiotherapy }]}{\text { [volume of hypoxic region before radiotherapy }]} \\
& =\exp \left(-\frac{\alpha}{\mathrm{OER}} d-\frac{\beta}{\mathrm{OER}^{2}} d^{2}\right)
\end{aligned}
$$

There is no consensus in the literature about how to modify the 'quadratic' component of cell death; however, the form used in Eq. (10) affords the interpretation of the OER as the multiplying factor for the dose escalation required under hypoxia in order to achieve the same cell kill as under normoxic conditions. We note that, in practice, this effect is likely to depend continuously on the local oxygen concentration. A continuous functional form for the OER (OER $=$ OER $(c)$ ) was proposed in Alper and Howard-Flanders (1956). For realistic parameter regimes, simulations using the continuous OER presented in Alper and Howard-Flanders (1956) and the discrete OER presented here did not significantly vary (results not shown). For these reasons, we restrict attention to the discrete OER stated above.

We assume further that dead material within the necrotic core is unaffected by radiation and so we impose that $R_{N}^{+}=R_{N}^{-}$.

Combining these assumptions, we deduce that, for a well-developed tumour spheroid (Fig. 2, case C3) with $0<R_{N}^{-}<R_{H}^{-}<R^{-}$, a dose $d$ of radiation gives a total volume change of

$$
\begin{aligned}
{[V]_{-}^{+}=} & \underbrace{\frac{4 \pi}{3}\left(R^{+^{3}}-R_{N}^{+^{3}}\right)}_{\text {viable volume after radiotherapy }}-\underbrace{\frac{4 \pi}{3}\left(R^{-3}-R_{N}^{-3}\right)}_{\text {viable volume before radiotherapy }} \\
& =-\underbrace{\frac{4 \pi}{3}\left(R^{-3}-R_{H}^{-3}\right)\left(1-\mathrm{sf}_{\text {normoxic }}\right)}_{\text {loss from proliferating rim }}-\underbrace{\frac{4 \pi}{3}\left(R_{H}^{-3}-R_{N}^{-3}\right)\left(1-\mathrm{sf}_{\text {hypoxic }}\right)}_{\text {loss from hypoxic region }},
\end{aligned}
$$




\section{Pre-radiotherapy}

\section{Post-radiotherapy}
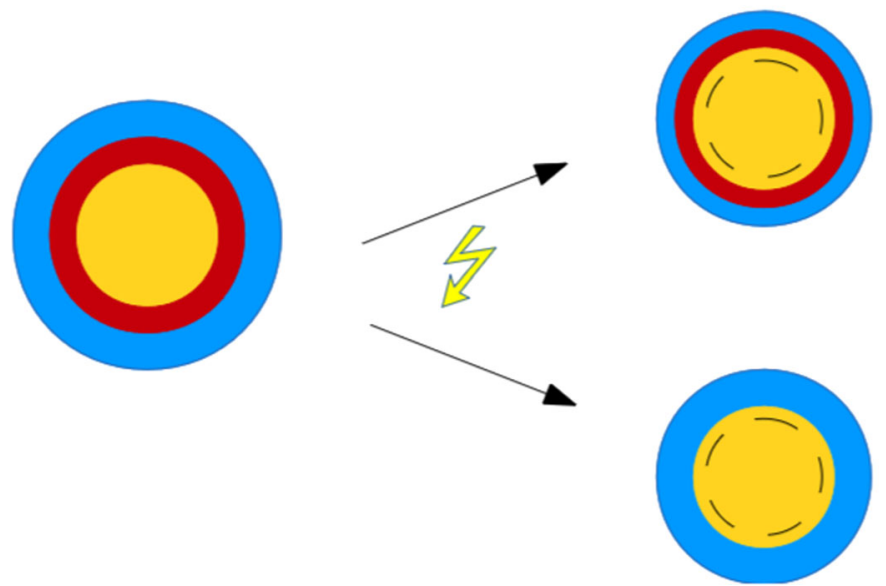

Fig. 3 Schematic of the model tumour spheroid post-radiotherapy. Dashed black line represents new position of the internal contour $c=c_{N}$ and shows the resulting mismatch between this contour and the boundary of the necrotic core, $R_{N}$. We note the absence of a hypoxic region in the bottom case

which, on rearrangement, yields the following expression for $R^{+}$in terms of $R^{-}, R_{H}^{-}$, and $R_{N}^{-}=R_{N}^{+}=R_{N}$ :

$$
R^{+^{3}}=\left(R^{-3}-R_{H}^{-3}\right) \mathrm{sf}_{\text {normoxic }}+\left(R_{H}^{-3}-R_{N}^{3}\right) \mathrm{sf}_{\text {hypoxic }}+R_{N}^{3} .
$$

The oxygen concentration profile associated with the new tumour structure can be determined [Eq. (2)] and $R_{H}$ defined implicitly as before [see Eq. (4)]. We note that while not all hypoxic tumour cells will be killed from a single radiotherapy fraction ( $f_{\text {hypoxic }}>0$ ), the instantaneous volume loss due to irradiation and the subsequent re-oxygenation of the tumour spheroid may result in a post-radiotherapy tumour composition without a hypoxic region.

Two cases can arise when a well-developed, 3-layer tumour is irradiated (see Fig. 3):

(i) $c_{N}<c\left(R_{N}^{+}\right)<c_{H}$-irradiated tumour is a fully developed tumour spheroid with 3 layers;

(ii) $c_{N}<c_{H}<c\left(R_{N}^{+}\right)$-irradiated tumour has a necrotic core, but no hypoxic annulus.

Since the necrotic core is assumed to be unaffected by radiotherapy, these are the only possible options. We have already described how a tumour spheroid of case (i) responds to radiation [Eq. (11)]. If a tumour with a pre-radiotherapy composition as in case (ii) is irradiated, then the corresponding volume change is given by

$$
[V]_{-}^{+}=-\frac{4 \pi}{3}\left(R^{-^{3}}-R_{N}^{3}\right)\left(1-\mathrm{sf}_{\text {normoxic }}\right)
$$

which, on rearrangement, yields the following expression for $R^{+}$in terms of $R^{-}$and $R_{N}$

$$
R^{+^{3}}=\left(R^{-^{3}}-R_{N}^{3}\right) \mathrm{sf}_{\text {normoxic }}+R_{N}^{3} .
$$




\subsubsection{Reconciling Pre- and Post-radiation Tumour Growth}

In the normal growth regime without radiotherapy, $R_{N}$ is defined implicitly by the oxygen concentration [see Eq. (5)]. When the tumour volume is reduced due to radiation, the oxygen concentration at the centre of the tumour increases and the location of the interface on which $c=c_{N}$ will shift towards the tumour centre or disappear. As the necrotic core is unaffected by radiation, $c\left(R_{N}^{+}\right)>c_{N}$ and the growth of the tumour spheroid immediately post-radiotherapy does not follow the original dynamics in the absence of treatment.

Between fractions of radiotherapy, repopulation of the tumour occurs. The tumour cells proliferate and die as before, however, while $c\left(R_{N}\right)>c_{N}$ no new material is added to the necrotic core, and so the existing necrotic material simply decays at the rate $\lambda_{A}+\lambda_{N}$. In this case, $R_{N}$ evolves according to

$$
R_{N}=R_{N}\left(t_{\text {fraction }}\right) \exp \left(-\frac{1}{3}\left(\lambda_{A}+\lambda_{N}\right)\left(t-t_{\text {fraction }}\right)\right)
$$

where $t_{\text {fraction }}$ is the time of the last fraction delivered for which $c\left(R_{N}\right)=c_{N}$, and $R_{N}\left(t_{\text {fraction }}\right)$ is the radius of the necrotic core upon irradiation. As such, prior to treatment with radiotherapy at $t=t_{\text {fraction }}$ the tumour composition is consistent with the Greenspan growth dynamics, while Eq. (13) governs the evolution of $R_{N}$ postirradiation.

Equations (2)-(4), (6)-(8) and (13) drive growth while $c\left(R_{N}\right)>c_{N}$, until the necrotic radius is such that $c\left(R_{N}\right)=c_{N}$, at which time the standard Greenspan model holds and growth is driven by Eqs. (2)-(8).

\subsection{Summary: Statement of Full Model (Dimensionless)}

The tumour growth model combined with fractionated radiotherapy (dose $d_{i}$ Gy delivered at times $\left.t=t_{i}, i=1,2, \ldots\right)$ can be summarised as follows:

$$
\begin{aligned}
& 0=\frac{1}{r^{2}} \frac{\partial}{\partial r}\left(r^{2} \frac{\partial c}{\partial r}\right)-\Gamma H\left(c-c_{N}\right) \\
& \frac{1}{4 \pi} \frac{\mathrm{d}}{\mathrm{d} t}\left(\frac{4 \pi R^{3}}{3}\right)=\int_{0}^{R}\left[c H\left(r-R_{H}\right)-\lambda_{A}-\lambda_{N} H\left(R_{N}-r\right)\right] r^{2} \mathrm{~d} r \\
& R_{H}=R_{N} \text { if } c>c_{H} \forall r \quad \text { and otherwise } c\left(R_{H}, t\right)=c_{H} \\
& R_{N}=R_{N}\left(t_{i}\right) \exp \left(-\frac{1}{3}\left(\lambda_{A}+\lambda_{N}\right)\left(t-t_{i}\right)\right) \quad \text { if } c\left(R_{N}\right)>c_{N} \text { and } t_{i}<t<t_{i+1}, \\
& R_{N}=0 \text { if } c>c_{N} \forall r \text { and } t<t_{i} \forall i, \quad c\left(R_{N}, t\right)=c_{N} \quad \text { otherwise } \\
& \frac{\partial c}{\partial r}=0 \text { at } r=0, \\
& c=c_{\infty} \text { at } r=R \\
& R(0)=R_{0} .
\end{aligned}
$$


Continuity conditions for $c$ and $\frac{\partial c}{\partial r}$ across $r=R_{H}, R_{N}$ are also imposed.

Radiotherapy, of dose $d_{i}$ applied at times $t=t_{i}$, effects an instantaneous volume change. The survival fraction of the normoxic tumour cell population is given by

$$
\mathrm{sf}_{\text {normoxic }}=\exp \left(-\alpha d_{i}-\beta d_{i}^{2}\right)
$$

while the survival fraction in hypoxic regions is given by

$$
\mathrm{sf}_{\text {hypoxic }}=\exp \left(-\frac{\alpha}{O E R} d_{i}-\frac{\beta}{O E R^{2}} d_{i}^{2}\right) .
$$

The necrotic core of the tumour spheroid is unaffected by radiotherapy. Immediately after a dose of radiation, the outer tumour radius is given by

$$
R^{+^{3}}=\left(R^{-^{3}}-R_{H}^{-{ }^{3}}\right) \mathrm{sf}_{\text {normoxic }}+\left(R_{H}^{-3}-R_{N}^{3}\right) \mathrm{sf}_{\text {hypoxic }}+R_{N}^{3} .
$$

NB: We subtly alter the equation for $R_{H}$ from the original Greenspan model so that $R_{H}=R_{N}$ if the threshold for hypoxia, $c_{H}$, is not reached. This does not change the dynamics in the case of untreated tumour growth, but ensures that the ordering of the tumour boundaries is conserved in the post-radiotherapy regime $\left(0 \leq R_{N} \leq R_{H} \leq R\right)$.

\section{Investigation of Model Behaviour}

We now consider the effects of various treatment protocols on a given tumour under this model. In Sect. 3.1, we solve the model numerically and highlight key features of the simulations for different parameter combinations. We explore these features in more detail in the following sections. In Sect. 3.2, we study tumour regrowth following a single dose of radiation, and in Sect. 3.3 we consider the long-term behaviour of a tumour for different fractionation schedules.

\subsection{Numerical Simulation Results}

For each tumour growth regime, the oxygen profile $c(r, t)$ may be solved analytically. Equations (14)-(23) may then be reduced to an ODE for $R$ and two algebraic equations for $R_{H}$ and $R_{N}$, as described in Appendix A.1. We solve the resulting system of equations numerically using a finite difference scheme implemented in MATLAB.

In Fig. 4a, we present results showing a typical tumour response to a conventional fractionation schedule ( 2 Gy, 5 days / week) simulated for 6 weeks using the parameter values in Tables 1 and 2. Cell death induced by the first fraction of radiation delivered at $t=1$ results in the loss of the hypoxic annulus. While $c\left(R_{N}\right)>c_{N}$, the necrotic core decays exponentially, as defined by Eq. (13). Figure 5a shows how the oxygen concentration in the necrotic core evolves throughout the first week of the radiotherapy protocol. We see the mismatch between $c\left(R_{N}\right)$ and $c_{N}$ following delivery of the first fraction (at $t=1$ ). As the tumour grows between fractions, oxygen concentration in 


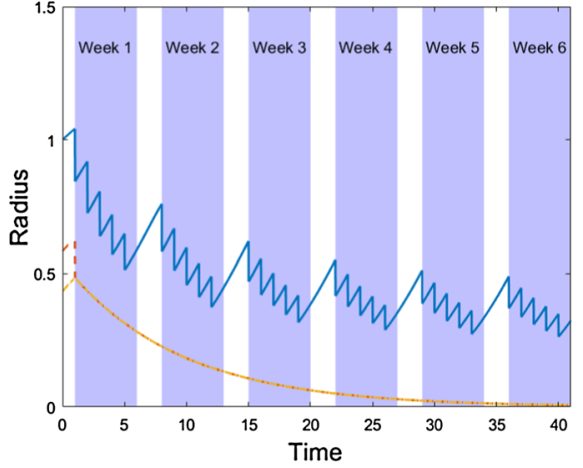

(a)

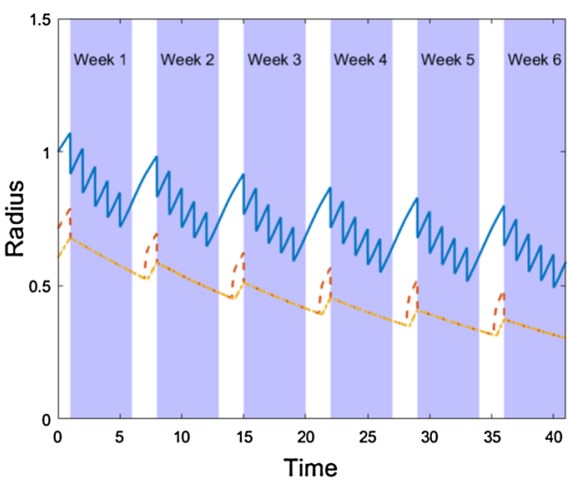

(b)

Fig. 4 Two solution of Eqs. (14)-(23) in response to a standard fractionation protocol (2 Gy fractions delivered daily Monday-Friday, shown as shaded regions) simulated for 6 weeks. Overall tumour radius, $R-$ blue solid line; hypoxic radius, $R_{H}$-red dashed line; radius of the necrotic core, $R_{N}$-yellow dot/dashed line. In $\mathbf{a}$, we use the parameter values given in Tables 1 and 2 with an initial tumour radius of 3 . In b, we simulate irradiating a tumour spheroid with lower rates of apoptosis $\left(\lambda_{A}=0.1213\right)$ and oxygen consumption $(\Gamma=0.3032)$

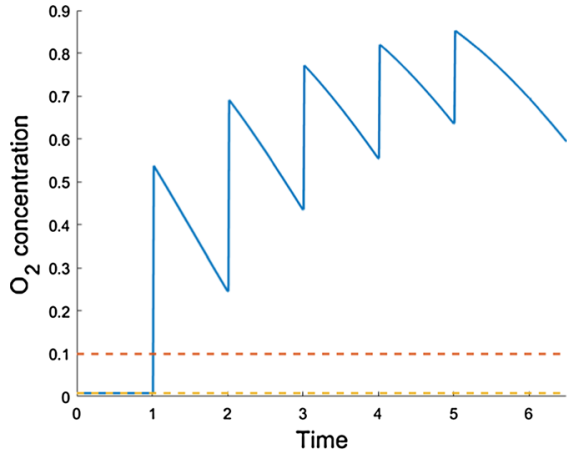

(a)

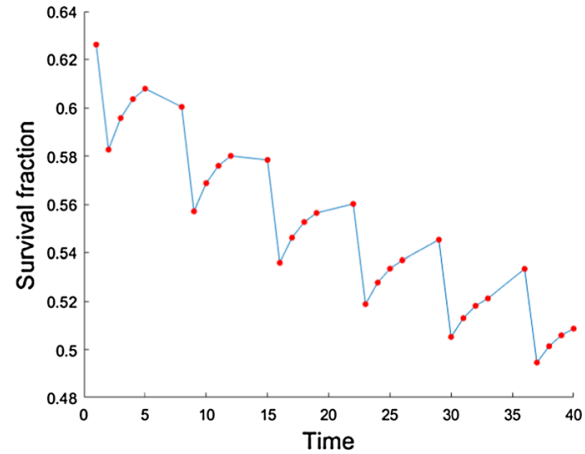

(b)

Fig. 5 Two plots highlighting key behaviours in the simulations shown in Fig. 4 using the same parameter values. a Blue line shows oxygen concentration (denoted $c$ in our model) within the necrotic core during the first week of treatment for the simulation in Fig. $4 \mathrm{a}$. The oxygen thresholds for hypoxia, $c_{H}$, and necrosis, $c_{N}$, are shown by dashed red and yellow lines, respectively. b Change in average tumour survival fraction following successive doses of radiotherapy corresponding to the simulation presented in Fig. 4b. Red points correspond to the survival fraction for each fraction of radiotherapy delivered, blue line added for ease of visualisation (NB. $y$-axis not $[0,1]$ )

the necrotic core decreases, while each fraction of radiation and the corresponding volume loss results in re-oxygenation.

For this parameter combination, radiotherapy results in a tumour volume at the end of treatment that is sufficiently small such that the spheroid is almost entirely composed of proliferative cells. In particular, the necrotic core has decayed so that $R_{N} \ll R$.

Figure $4 \mathrm{~b}$ shows how the system dynamics change when a tumour with a much lower apoptotic rate $\left(\lambda_{A}\right)$ is exposed to the standard fractionation protocol. The slower 
rate of apoptosis results in a larger steady state tumour in the absence of radiotherapy. Similarly, a larger tumour volume is supported throughout treatment and a more gradual volume reduction is observed. In this case, the hypoxic annulus reappears during treatment (as observed during the weekend breaks in the protocol) since the necrotic core decays such that $c\left(R_{N}\right)=c_{H}$ before the end of the fractionation protocol. A rapid transient increase in $R_{H}$ is observed when the hypoxic annulus reappears. We characterise this behaviour in Appendix A.3 where we show that $R_{H}$ increases like $\sqrt{t}$ on this short timescale.

For the simulation in Fig. 4b, a tumour spheroid comprising a necrotic core and transient hypoxia persists at the end of treatment. The tumour volume appears to be evolving towards a periodic orbit and, as such, the model predicts that continuing the same fractionation schedule indefinitely will not yield significant further volume reduction.

Since the tumour composition changes dynamically throughout treatment, the efficacy of each radiation fraction also varies. The survival fraction changes by about $10 \%$ from the start to the end of treatment due to the shrinkage of the necrotic core (Fig. 4b). In this case, a characteristic shape within the curve in Fig. 5b is repeated weekly due to the weekly oscillations in tumour composition, with the spike associated with the rapid reappearance of hypoxia. Such dynamic behaviour clearly depends on tumour-specific parameters and highlights the additional details that can be observed when spatial effects are included in a mathematical model (c.f. difference in tumour composition between Fig. 4a, b).

\subsection{Single Hit Regrowth}

We now consider the effect of irradiating a small tumour spheroid composed entirely of proliferating cells and its subsequent regrowth. This situation is relevant for tumours of radius $R$ such that $0<R^{2}<\frac{6}{\Gamma}\left(c_{\infty}-c_{H}\right)$ and $R_{H}=R_{N}=0$, since when $R=\sqrt{\frac{6}{\Gamma}\left(c_{\infty}-c_{H}\right)}, c(0)=c_{H}$ and therefore larger tumours will contain a hypoxic region and/or necrotic core. Solving Eq. (14) subject to boundary conditions (18)-(19), we deduce that the oxygen profile for this tumour composition is given by

$$
c(r, t)=c_{\infty}-\frac{\Gamma}{6}\left(R^{2}-r^{2}\right) .
$$

Substituting from Eq. (24) into Eq. (15), with $R_{H}=R_{N}=0$, we arrive at the following ODE for $R(t)$ :

$$
\frac{\mathrm{d} R}{\mathrm{~d} t}=\frac{R}{3}\left(c_{\infty}-\frac{\Gamma R^{2}}{15}-\lambda_{A}\right)
$$

with solution

$$
\frac{R}{R_{0}}=\sqrt{\frac{c_{\infty}-\lambda_{A}}{\frac{\Gamma R_{0}^{2}}{15}+A_{0} e^{-\frac{2}{3}\left(c_{\infty}-\lambda_{A}\right) t}}}
$$


where $A_{0}=c_{\infty}-\lambda_{A}-\frac{\Gamma R_{0}^{2}}{15}$.

We can use Eq. (26) to calculate the time taken for a tumour spheroid of radius $0<\tilde{R}^{2}<\frac{6}{\Gamma}\left(c_{\infty}-c_{H}\right)$ following a single fraction of radiation of dose $d$ to regrow to its initial (i.e. pre-radiotherapy) size. The tumour radius immediately after irradiation is given by $\gamma(d) \tilde{R}$, where $\gamma(d)=\exp \left(-\frac{1}{3}\left(\alpha d+\beta d^{2}\right)\right)$ is the survival fraction given by the linear-quadratic formula. It is straightforward to show that the time, $\Delta t$, until the tumour regrows to its original size is given by

$$
\Delta t=\frac{3}{2\left(c_{\infty}-\lambda_{A}\right)} \ln \left[\frac{c_{\infty}-\lambda_{A}-\frac{\gamma^{2} \Gamma \tilde{R}^{2}}{15}}{\gamma^{2}\left(c_{\infty}-\lambda_{A}-\frac{\Gamma \tilde{R}^{2}}{15}\right)}\right],
$$

where $\gamma=\gamma(d)$. We assume that the parameters are such that the tumour spheroid was growing pre-irradiation and therefore $R^{2}<\frac{15}{\Gamma}\left(c_{\infty}-\lambda_{A}\right)$ [from (25)]. In this parameter regime, the logarithm in Eq. (27) is defined and $\Delta t>0$. By extension, we require that $c_{\infty}>\lambda_{A}$ since otherwise the tumour spheroid shrinks for all values of $\tilde{R}$ and a viable tumour of any size cannot be supported. We note for future reference that

$$
\Delta t \rightarrow \frac{\alpha d+\beta d^{2}}{c_{\infty}-\lambda_{A}} \quad \text { as } \quad \tilde{R} \rightarrow 0 .
$$

Differentiating Eq. (27) with respect to $\tilde{R}$, we obtain

$$
\frac{\mathrm{d}}{\mathrm{d} \tilde{R}}(\Delta t)=\frac{\Gamma \tilde{R}}{5} \frac{\left(1-\gamma^{2}\right)}{\left(c_{\infty}-\lambda_{A}-\frac{\Gamma \tilde{R}^{2}}{15}\right)\left(c_{\infty}-\lambda_{A}-\gamma^{2} \frac{\Gamma \tilde{R}^{2}}{15}\right)} .
$$

Therefore, at least for small $\tilde{R},\left.\frac{\mathrm{d} \Delta t}{\mathrm{~d} \tilde{R}}\right|_{\gamma(d)}>0$, and so the regrowth time, $\Delta t$, is an increasing function of $\tilde{R}$ for fixed dose $d$. This holds for all $0<\tilde{R}^{2}<\frac{6}{\Gamma}\left(c_{\infty}-c_{H}\right)$ if the inequality $3 c_{\infty}-5 \lambda_{A}+2 c_{H}>0$ is satisfied. Then, Eq. (27) represents an increasing family of curves where the minimum bounding curve is given by Eq. (28). That is, for $0<\tilde{R}_{1}<\tilde{R}_{2}<\sqrt{\frac{6}{\Gamma}\left(c_{\infty}-c_{H}\right)}$ and fixed dose $d, \Delta t\left(d ; R_{1}\right)<\Delta t\left(d ; R_{2}\right)$.

Returning to Eq. (27), we see that for an initial radius $\tilde{R}$, a strategy that combines a dose $d$ with an inter-fraction time less than $\Delta t$ will result initially in a net shrinkage of the tumour throughout treatment (i.e. the tumour volume is smaller at the delivery of each radiation fraction). Conversely, if we wait longer than $\Delta t$ to re-irradiate, then the tumour will have grown larger than its original size. Thus, Eq. (27) defines a curve in the $(d, \Delta t)$-plane for treatment protocols which give rise to periodic behaviour for a given initial tumour radius $\tilde{R}$, since the cell kill induced by radiotherapy is exactly balanced by the regrowth between fractions. This curve is indicated in Fig. 6. We note that the curve describing protocols that yield periodic behaviour becomes concave for large $R$, and in particular, as $R$ tends to its steady state value, $R^{*}$ say, then for a given dose, $d>0, \Delta t(d) \rightarrow \infty$. 

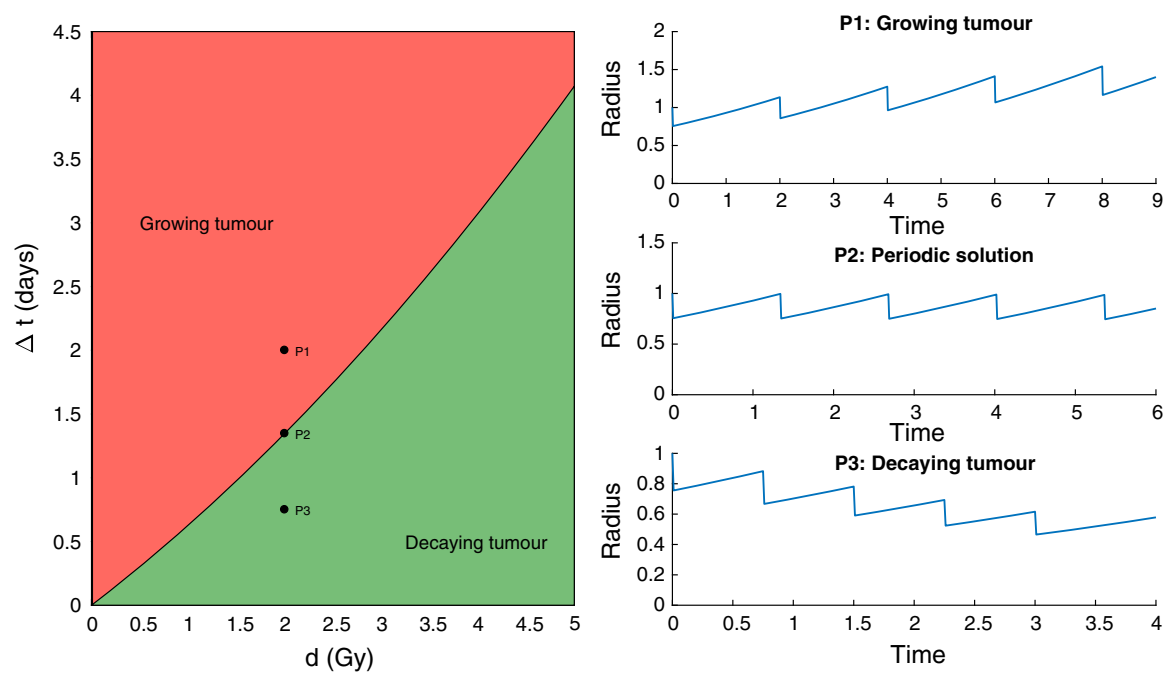

Fig. 6 Radiation dose and fractionation-dependent behaviour for a tumour spheroid composed of proliferating cells. Line for periodic behaviour given by Eq. (27). Plots on the right show response to 5 fractions of radiotherapy given by the protocols corresponding to points P1, P2 and P3.

\subsection{Periodic Surface in 'Treatment Space'}

In the previous section, we investigated the response of tumours of fixed initial radius $\tilde{R}$ to different fractionation protocols as specified by a dose, $d$, and inter-fraction time, $\Delta t$. The $(d, \Delta t)$-plane can be partitioned into regions in which the tumour volume increases or decreases during treatment. However, we note that the location of these regions depends on the tumour radius at the time of irradiation, $\tilde{R}$. As such, when considering the behaviour of a tumour throughout an entire course of radiotherapy, it is not a line in the $(d, \Delta t)$-plane that we are interested in, but a surface in 'treatment space', $\mathbb{T} \subset \mathbb{R}^{3}$, with components $(R, d, \Delta t)$.

For a protocol delivering $n$ fractions of radiation, we can identify the response of a given tumour with a discrete trajectory, $\left(R\left(t_{i}\right), d_{i}, \Delta t_{i}\right) \in \mathbb{T}$, through treatment space. Note, we consider the trajectory as discrete points pre-irradiation so that $R\left(t_{i}\right)=$ $R\left(t_{i}\right)^{-}$in our previous notation. Here, $t_{i}, d_{i}$ and $\Delta t_{i}$ are the time, dose and inter-fraction time, respectively, of the $i$ th fraction, for $i=1, \ldots, n$. The union of the curves in the $(d, \Delta t)$-plane determining periodicity defines a surface, $\mathrm{S}$, in $\mathbb{T}$ (see Fig. 7a). Now, as a tumour progresses through the course of radiotherapy, points on its trajectory that lie above this surface indicate net growth of the tumour by the time of delivery of the next radiation dose, while points below the surface result in a more desirable decrease in tumour volume. Therefore, for a given tumour spheroid, $\mathrm{S}$ defines a surface that partitions $\mathbb{T}$ into treatment protocols that cannot halt tumour progression and those that lead to tumour decay.

With this understanding, more general statements about the outcome of different fractionation protocols can be made. We first consider dosing schedules in which the same dose $d$ is administered at constant intervals $\Delta t$. In this case, if a point in $\mathbb{T}$ lies 


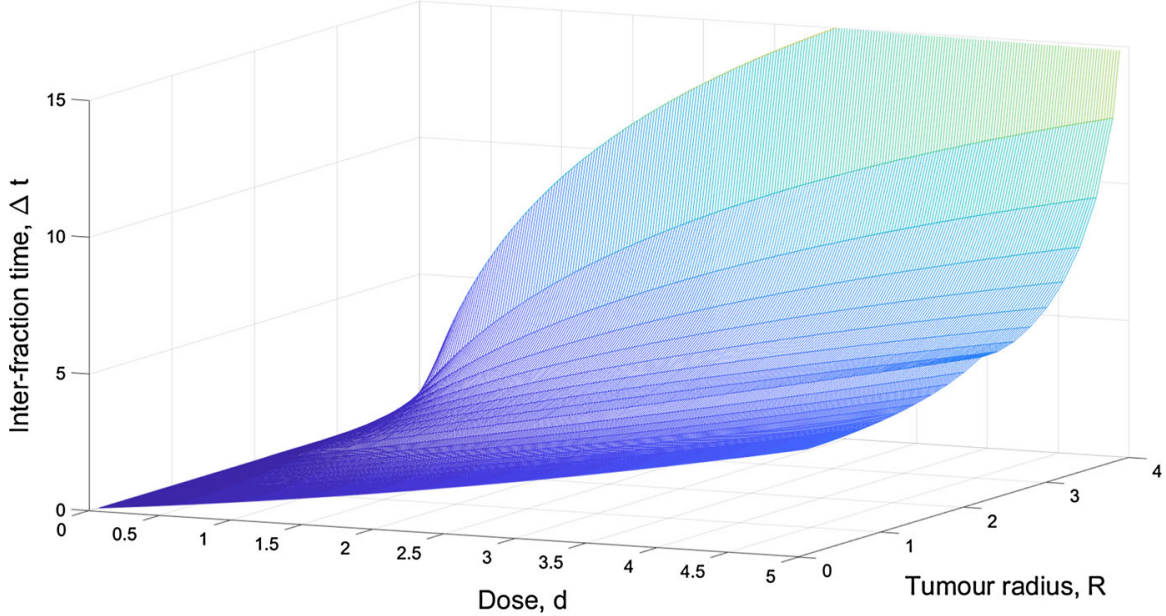

(a)

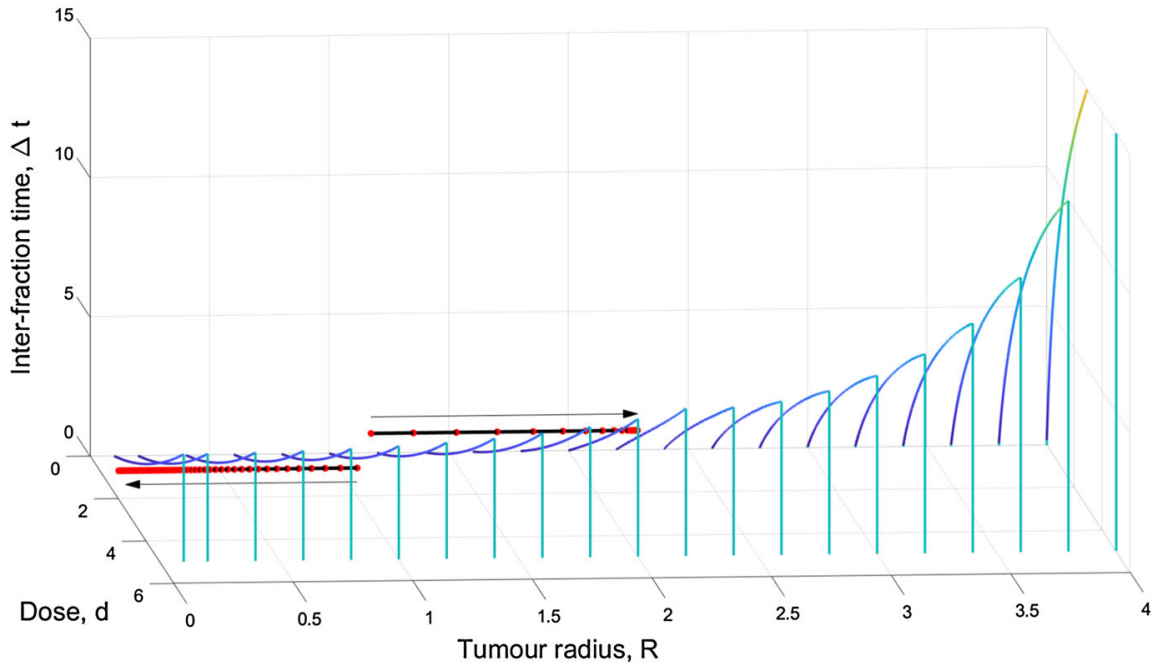

(b)

Fig. 7 a Surface, $S$, corresponding to protocols giving rise to periodic treatment responses. b Simulated trajectories (black lines) in 'treatment space', $\mathbb{T} \subset \mathbb{R}^{3}$ with components $(R, d, \Delta t)$, for two different constant dosing schedules (fractions plotted as red points) applied to the same tumour spheroid (i.e. same model parameters). A dose of 3 Gy every 3 days (top trajectory) results in a tumour that grows until it reaches a periodic state, while a dose of 2 Gy delivered daily (bottom trajectory) is sufficient to drive the tumour volume arbitrarily close to zero. The surface describes treatment protocols which give rise to periodic behaviour

above $\mathrm{S}$, then the tumour spheroid will grow until it converges to a point on $\mathrm{S}$, at which time it becomes periodic (Fig. 7b).

Below $\mathrm{S}$, the outcome of radiotherapy depends on the boundary curve of $\mathrm{S}$ as $R \rightarrow 0$ [see Eq. (28)]. If the fractionation schedule lies above this curve in the $(d, \Delta t)$-plane, then the system will eventually converge to the corresponding point on S. However, for $d$ and $\Delta t$ values below this curve the tumour decays to arbitrarily small volumes 
(Fig. 7b). We note that Eq. (28) still holds for this analysis even though its derivation requires an initial tumour composed entirely of viable cells. This is the case since, in the model, necrotic material is only created via nutrient starvation and not as a result of irradiation. As such, for ongoing treatment protocols that result in a decreasing tumour volume, $R \gg R_{N}$ as $t \rightarrow \infty$.

We can extend this concept to more general treatment schedules. If there exists $m \in \mathbb{N}$ such that for all $i>m$ the point $\left(d_{i}, \Delta t_{i}\right)$ of the $i$ th fraction lies below the curve given by Eq. (28), then treatment is sufficient to drive the tumour volume arbitrarily close to 0 . However, using Fig. $4 \mathrm{~b}$ as an illustrative example, we observe a simulation in which the weekend break of the standard fractionation protocol corresponding to $\Delta t=3$ days gives rise to the periodic orbit.

When predicting how a tumour may respond to radiotherapy, two quantities of interest are the potential volume doubling time, $T_{\text {pot }}$, and the survival fraction after 2 Gy of radiation, $S_{2}$ Gy, of the tumour. We translate these definitions into the model and identify tumour characteristics for which the model would eventually predict regrowth over the weekend.

The survival fraction at $2 \mathrm{~Gy}, S_{2 \mathrm{~Gy}}$, for normoxic tumour cells is given by the linear-quadratic formulation. In order to find an expression for $T_{\text {pot }}$, we consider the tumour dynamics for small tumour volumes that are in an 'exponential growth' phase. Linearising the model equations for small $R$, we obtain

$$
\frac{\mathrm{d} R}{\mathrm{~d} t} \sim\left(c_{\infty}-\lambda_{A}\right) \frac{R}{3}
$$

which yields a volume doubling time of

$$
T_{\text {pot }}=\frac{\ln 2}{c_{\infty}-\lambda_{A}},
$$

where the factor of $\frac{1}{3}$ disappears when we convert from tumour radius to tumour volume. Substituting these expressions into Eq. (28) for a standard 2 Gy dose, we obtain

$$
\Delta t=-\ln \left(S_{2 G y}\right) \frac{T_{\mathrm{pot}}}{\ln 2} .
$$

We note that this expression holds for tumour growth models in which the growth of small tumours is exponential and radiation-induced cell death is modelled as an instantaneous volume loss.

If we now consider Eq. (30) in the context of the weekend break $(\Delta t=3)$ within the standard fractionation protocol, then we see that $-T_{\text {pot }} \ln \left(S_{2 \text { Gy }}\right)<3 \ln 2$ defines a class of tumour characteristics for which regrowth of the tumour over the weekends impedes tumour decay (Fig. 8).

\section{Discussion}

In vivo tumours are highly heterogeneous cellular entities characterised by high inter-patient variability. Allied to this, the local efficacy of radiotherapy delivered 


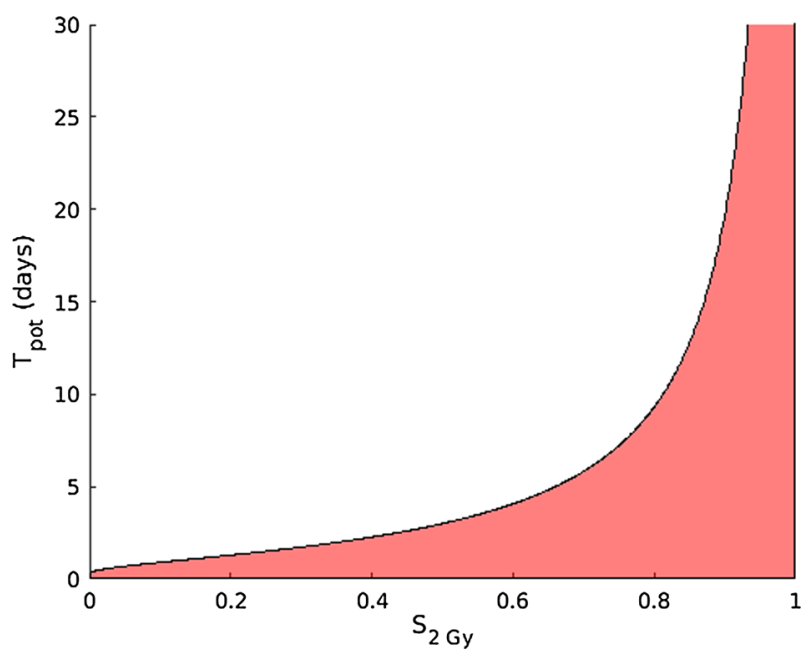

Fig. 8 Region for which tumours will eventually exhibit net growth over the weekend (shown in red), with the bounding curve given by Eq. (30) for $\Delta t=3$

to the tumour site is affected by a number of variables associated with the tumour's heterogeneous composition and micro-environment. In particular, the local oxygen concentration can significantly influence radiation-induced cell death, with welloxygenated regions being shown to exhibit up to threefold greater radiosensitivity than hypoxic tumour populations. With this in mind, in this paper we have presented a simple, spatially resolved model in order to investigate the effects of tumour composition on radiotherapy response. Our model builds on the tumour growth model first proposed by Greenspan (1972). We extend this to incorporate the effects of radiotherapy taking into account spatially varying radiosensitivities.

Numerical simulations and mathematical analysis of the model reveal how the tumour's growth dynamics and spatial composition change throughout treatment. Heterogeneity within the tumour not only affects the initial response to radiotherapy, but also how this response changes throughout the duration of the treatment protocol (see Fig. 5b). For parameter regimes in which hypoxia re-emerges during treatment, a rapid transient increase in the width of the hypoxic annulus is observed. This behaviour arises naturally from the model and the underlying process driving this phenomenon remains to be elucidated. The model more generally also classifies protocols that may result in tumour progression, a nonzero periodic tumour volume, or tumour decay. We identify a surface in 'treatment space' dependent on tumour-specific growth and radiosensitivity parameters and determine that successful protocols correspond to those that remain below this surface throughout treatment. The wide variety of dynamics observed suggests that spatial heterogeneity may be important for simulating tumour response to radiotherapy and, in particular, for making clinical predictions.

The model presented in this paper makes numerous assumptions and simplifications about the underlying biology. For radiation-induced cell death, we take the common approach of modelling this process as an instantaneous effect. However, the linearquadratic model was established to determine long-term clonogenic survival after 
radiotherapy. Biologically, cell death after radiation may occur via a number of different mechanisms, with many irradiated cells dying only after attempting mitosis one or more times (Joiner and van der Kogel 2009). Consequently, radiation-induced cell death may not elicit the instantaneous volume reduction modelled here. In future work, we will model this process in more detail in order to describe the short term response to radiotherapy and the corresponding spatial changes in tumour composition more accurately. Additionally, a more detailed model will allow us to relax the restrictions of the spherically symmetric geometry associated with the current model, making it more applicable to modelling vascular tumour growth and in vivo responses to radiotherapy.

Radiation biology has a rich history of integrating theoretical and quantitative approaches to advance biological studies and clinical concepts. From the simple and widely used LQ model emerged the concepts of biologically equivalent dose (BED) and tumour control probability (TCP) to guide the development of clinical fractionation protocols. Different TCP models with increasing complexity, from simple Poissonian TCP to stochastic birth-death processes, have recently been scrutinised, and mathematical analysis suggests that simple models may be best positioned for clinical utility (Gong et al. 2013). Clinical data are often insufficient to inform mathematical models (Chvetsov et al. 2015). Both simple and more complex mathematical models are facing numerous hurdles in the attempt to integrate them into radiation oncology, and more work is needed to fully harness the potential of mathematical modelling for precision radiation oncology (Rockne and Frankel 2017).

However, for a model to be useful in making patient-specific predictions in the clinic, parameters must be identifiable with respect to the limited clinical data typically available. As such, current research in this area often focusses on phenomenological ODE models with few parameters to be estimated. Typically, these models contain no information about the spatial heterogeneity in tumour composition and radiotherapy response. With this in mind, we also propose a comparison of the developed spatially resolved model with these phenomenological approaches as future work. We aim to identify situations in which the spatially resolved and spatially averaged models agree well, and those in which there is a significant difference. The tumour composition changes observed in model simulations suggests that averaged parameter values in simple, phenomenological models may not sufficiently capture the tumour dynamics during treatment for some tumour compositions and parameter combinations.

While more sophisticated models may be difficult to parametrise in practice, they have the potential to increase biological insight and inform further modelling studies. Spatially resolved models, such as the one presented in this paper and the future work proposed to generalise some of the simplifying assumptions made here, may aid in the development of alternative clinically focussed models which capture more of the key features than existing phenomenological models. More complex models incorporating more biological detail may also be used to generate data for in silico testing of ODE models and model selection paradigms; comparing the quality of fit and future predictions of a range of simple models against a known 'ground-truth'. 
Acknowledgements This work was supported by the Engineering and Physical Sciences Research Council [grant number EP/G037280/1]. TL would also like to thank the Moffitt Cancer Center, where some of this work was undertaken, for their hospitality.

Open Access This article is distributed under the terms of the Creative Commons Attribution 4.0 International License (http://creativecommons.org/licenses/by/4.0/), which permits unrestricted use, distribution, and reproduction in any medium, provided you give appropriate credit to the original author(s) and the source, provide a link to the Creative Commons license, and indicate if changes were made.

\section{Appendix}

\section{A.1 Explanation of Greenspan's Original Growth Model}

Here, we provide further explanation of Greenspan's original model for the nutrientlimited growth of tumour spheroids (Greenspan 1972). We restate the equations for the evolution of the nutrient concentration profile, $c$, and the tumour radii, $R, R_{H}$ and $R_{N}$.

$$
\begin{aligned}
& 0=\underbrace{\frac{1}{r^{2}} \frac{\partial}{\partial r}\left(r^{2} \frac{\partial c}{\partial r}\right)}_{\text {diffusion term }}-\underbrace{\Gamma H\left(c-c_{N}\right)}_{\text {oxygen consumption }}, \\
& \underbrace{\frac{1}{4 \pi} \frac{\mathrm{d}}{\mathrm{d} t}\left(\frac{4 \pi R^{3}}{3}\right)}_{\text {rate of change of tumour volume }}=\int_{0}^{R}[\underbrace{c H\left(c-c_{H}\right)}_{\text {cell proliferation term }}-\underbrace{\lambda_{A}-\lambda_{N} H\left(c_{N}-c\right)}_{\text {cell death term }}] r^{2} \mathrm{~d} r,
\end{aligned}
$$

$$
\begin{aligned}
& R_{H}=0 \text { if } c>c_{H} \forall r \text { and otherwise } c\left(R_{H}, t\right)=c_{H}, \\
& R_{N}=0 \text { if } c>c_{N} \forall r \text { and otherwise } c\left(R_{N}, t\right)=c_{N}, \\
& \frac{\partial c}{\partial r}=0 \text { at } r=0, \\
& c=c_{\infty} \text { at } r=R \\
& R(0)=R_{0} .
\end{aligned}
$$

In (31), we assume that the oxygen concentration within the tumour is regulated by its diffusion across the tumour (with diffusion constant $D$ ) and consumption by the tumour cells. We make the further assumption that both normoxic and hypoxic cells consume oxygen at the same constant rate, $\Gamma$, and that cells in the necrotic core do not consume oxygen. Since oxygen diffuses on a much shorter timescale than tumour growth we assume that the oxygen concentration is in a quasi-steady state. Hence, $c(r, t)$ satisfies (31), where $H($.$) is the Heaviside function, with associated boundary$ conditions (35) and (36) imposing symmetry at $r=0$ and a Dirichlet boundary condition at $r=R(t)$.

We assume that rates of cell proliferation and death within the tumour are determined by the local oxygen concentration. Proliferation occurs at a rate proportional 
to $c$ where there is sufficient oxygen supply $\left(c>c_{H}\right)$. Both apoptosis and necrosis contribute to cell death within the tumour, with apoptosis occurring at a constant rate throughout the tumour and necrosis localised to the necrotic core (where $c<c_{N}$ ). Degradation of the necrotic core is assumed to result in material that is freely permeable throughout the tumour spheroid. We assume that adhesion and surface tension forces acting on the tumour cells maintain the shape of the tumour spheroid and that these same forces push cells inwards to compensate for the outward flux of necrotic material from the necrotic core. The evolution of $R(t)$ is then given by the mass balance in Eq. (32), with $\lambda_{A}, \lambda_{N}>0$, accompanied by the initial condition (37).

The radii at which the tumour becomes hypoxic and necrotic, $R_{H}$ and $R_{N}$, respectively, are determined as contours of the oxygen concentration profile [Eqs. (33) and (34)]. For situations in which the tumour spheroid is small enough that one or both of these contours do not exist, we define the corresponding radius to be 0 .

An analytic solution can be found for $c(r, t)$ that depends on $R, R_{H}$ and $R_{N}$, and so this system can be reduced to an ODE for $R$ and algebraic equations for $R_{H}$ and $R_{N}$. The resulting equations in the case of a fully developed, 3-layer tumour spheroid are given in Eq. (38)-(42). The system of equations for the earlier growth phases is similar and can be obtained in the same manner.

$$
\begin{gathered}
c= \begin{cases}c_{N} & 0<r<R_{N} \\
c_{N}+\frac{\Gamma}{6 r}\left(r-R_{N}\right)^{2}\left(r+2 R_{N}\right) & R_{N}<r<R,\end{cases} \\
\frac{\mathrm{d} R}{\mathrm{~d} t}=\frac{R}{3}\left[c_{N}\left(1-\frac{R_{H}^{3}}{R^{3}}\right)-\left(\lambda_{A}+\lambda_{N} \frac{R_{N}^{3}}{R^{3}}\right)\right] \\
\quad+\frac{\Gamma R^{3}}{6}\left[\frac{1}{5}\left(1-\frac{R_{H}^{5}}{R^{5}}\right)-\frac{R_{N}^{2}}{R^{2}}\left(1-\frac{R_{H}^{3}}{R^{3}}\right)\right. \\
\left.+\frac{R_{N}^{3}}{R^{3}}\left(1-\frac{R_{H}^{2}}{R^{2}}\right)\right], \\
\left(1-\frac{R_{N}}{R}\right)^{2}\left(1+\frac{2 R_{N}}{R}\right)=\frac{6}{\Gamma R^{2}}\left(c_{\infty}-c_{N}\right), \\
\left(1-\frac{R_{N}}{R_{H}}\right)^{2}\left(1+\frac{2 R_{N}}{R_{H}}\right)=\frac{6}{\Gamma R_{H}^{2}}\left(c_{H}-c_{N}\right), \\
R(0)=R_{0} .
\end{gathered}
$$

Figure 2 (main text) demonstrates the growth and various stages (labelled C1, C2 and C3) of tumour spheroid composition under the Greenspan model using the parameter values in Table 3. In using partial pressures of oxygen rather than concentration, we follow Grimes et al. (2014) and use Henry's law to convert between the two, so that $p=\Omega c$, with $\Omega=3.0318 \times 10^{7} \mathrm{mmHg} \mathrm{kg} \mathrm{m}^{-3}$. The oxygen concentration profile at each time point is a monotonic function increasing outwards from the tumour centre. Initially, for very small, avascular tumour spheroids, the entire 
Table 3 Parameter values for Greenspan's model of tumour spheroid growth

\begin{tabular}{llll}
\hline Parameter & Symbol & Value & Source \\
\hline Oxygen consumption rate $\left(\mathrm{m}^{3} \mathrm{~kg}^{-1} \mathrm{~s}^{-1}\right)$ & $\Gamma$ & $7.29 \times 10^{-7}$ & Grimes et al. (2014) \\
Partial pressure at tumour boundary $(\mathrm{mmHg})$ & $p_{\infty}$ & 100 & Grimes et al. (2014) \\
Hypoxic partial pressure $(\mathrm{mmHg})$ & $p_{H}$ & 10 & Grimes et al. (2014) \\
Anoxic partial pressure $(\mathrm{mmHg})$ & $p_{N}$ & 0.8 & Grimes et al. (2014) \\
Oxygen diffusion constant $\left(\mathrm{m}^{2} \mathrm{~s}^{-1}\right)$ & $D$ & $2 \times 10^{-9}$ & Grimes et al. (2014) \\
Apoptosis constant $\left(\mathrm{m}^{3} \mathrm{~kg}^{-1}\right)$ & $\lambda_{A}$ & $1.0555 \times 10^{-6}$ & Frieboes et al. (2007) \\
Necrosis constant $\left(\mathrm{m}^{3} \mathrm{~kg}^{-1}\right)$ & $\lambda_{N}$ & $2 \times 10^{-8}$ & Schaller and Meyer-Hermann \\
& & & (2006) \\
Cell birth/death constant $\left(\mathrm{kg} \mathrm{m}^{-3} \mathrm{~s}^{-1}\right)$ & $s$ & 3.509 & Frieboes et al. (2007) \\
\hline
\end{tabular}

Dimensional apoptosis and necrosis rates, $\mathrm{s}^{-1}$, are given by $s \lambda_{A}$ and $s \lambda_{N}$, respectively. $p_{\infty}, p_{H}$ and $p_{N}$ are partial pressures which, for consistency, are then converted into concentrations $c_{\infty}, c_{H}$ and $c_{N}$, respectively. For further details, see Appendix A.1

tumour is made up of viable, proliferating cells (case C1). We notice that as the tumour grows and so the oxygen concentration at the centre of the tumour decreases, a hypoxic region within the tumour begins to develop (case C2) followed by a central necrotic core when the oxygen concentration falls so low as to be unable to support viable cells (case C3). These different regions, and their varying responses to radiation, require careful consideration when extending Greenspan's model to account for radiotherapy. Further discussion of the different phases of growth is given in Byrne (2012).

\section{A.2 Parameter Sweep of Greenspan Growth Dynamics}

We initially investigate the sensitivity of the standard Greenspan growth dynamics to some of its key parameters. In particular, as we sweep over a region of parameter space, we observe the resulting long-time, steady state properties of the tumour spheroids. Specifically, we investigate how the availability of oxygen, $c_{\infty}$, the oxygen consumption rate of the cells, $\Gamma$, and the rates of apoptosis and necrosis, $\lambda_{A}$ and $\lambda_{N}$, respectively, affect the growth of the tumour under this model. We choose appropriate intervals for each parameter, in each case encompassing the corresponding value shown in Table 3, and systematically explore the resulting region of parameter space, solving the model numerically. We plot heatmaps in parameter space in order to observe the behaviour of a variety of quantities of interest of the tumours at steady state (Figs. 9, 10). We have omitted here the results of varying both $c_{\infty}$ and $\lambda_{N}$ since, in the case of $c_{\infty}$, increasing the parameter value simply has the effect of an increase in overall tumour size and the width of the corresponding proliferating rim, as might be expected, while, for the range of values swept over, $\lambda_{N}$ had little effect on the observed tumour characteristics.

Broadly speaking, we see in Fig. 9 that relatively increasing the amount of oxygen available to the tumour cells (by decreasing the consumption rate $\Gamma$ ), or decreasing 

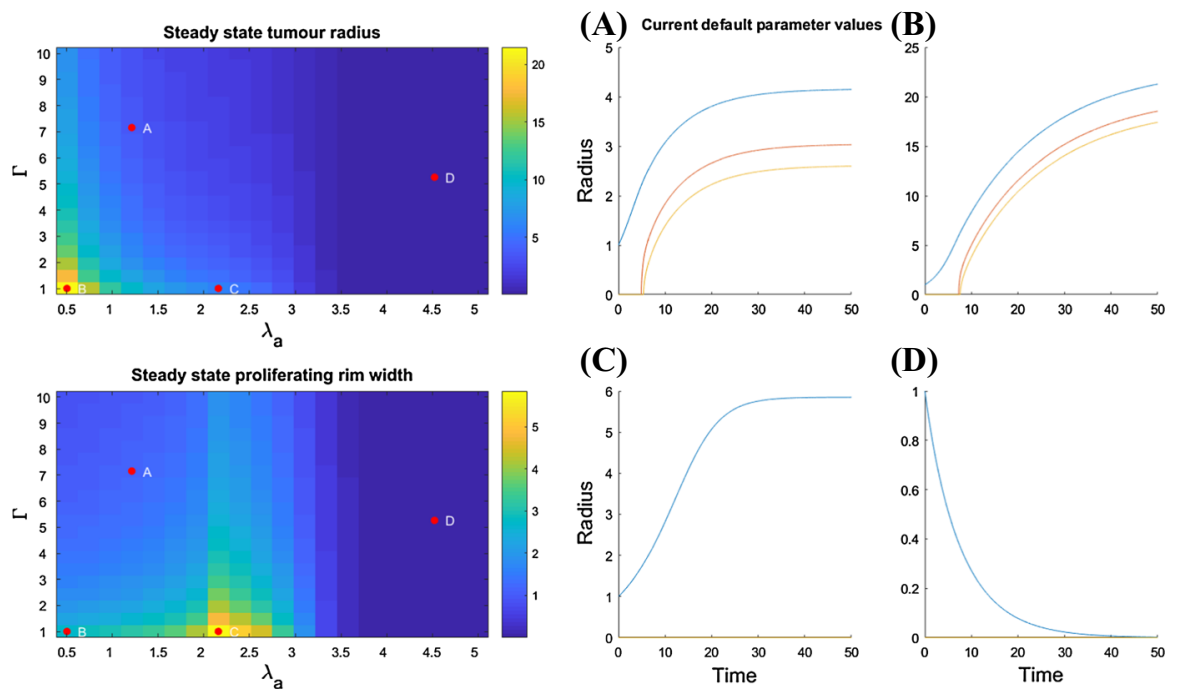

Fig. 9 Results of a parameter sweep for the steady state tumour radius and width of proliferating rim with corresponding tumour growth profiles at points of interest in parameter space, A, B, C and D, as given by the numerical solutions to Eqs. (2)-(8)
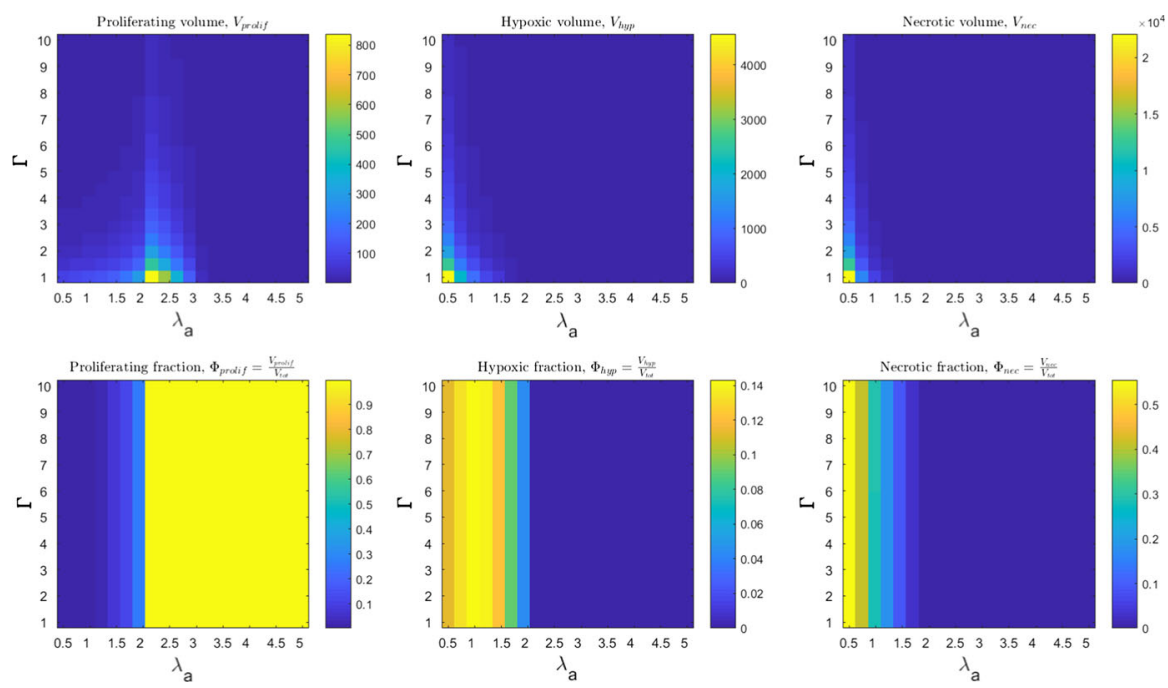

Fig. 10 Heatmaps for the steady state volumes of proliferating, hypoxic and necrotic cells, and the corresponding volume fractions

the death rate, $\lambda_{A}$, results in larger steady state tumours, as we might expect. Point A marks the location of the parameter values from Table 3. Extreme points in the region of concern in parameter space are labelled B, C and D, and the corresponding tumour evolution profiles shown. We see that the greater relative availability of oxygen and the low death rate at point B results in a large, fully developed tumour spheroid, whereas 


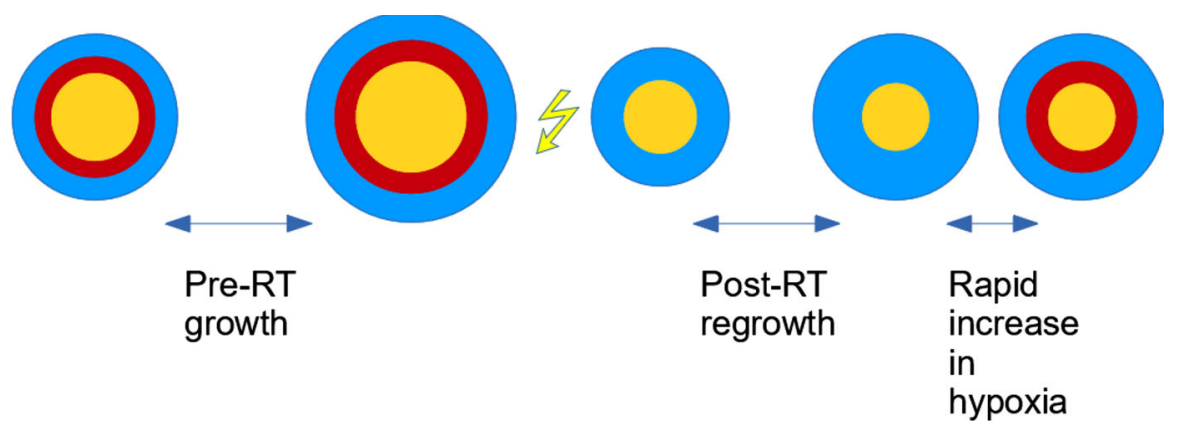

Fig. 11 Schematic contextualising the scenario in which the rapid transient increase in hypoxia is observed, corresponding to the different growth phases observed in Fig. 12

the high rate of apoptosis at $\mathrm{D}$ is such that a viable, tumour cell population cannot be sustained.

For a given $\Gamma$, we can determine the onset of necrosis as the radius $\bar{R}=$ $\sqrt{\frac{6}{\Gamma}\left(c_{\infty}-c_{N}\right)}$. At the point $\mathrm{C}$, and any other point on a vertical line through $\mathrm{C}$, the rate of apoptosis is such that the tumour reaches a steady state of radius $\bar{R}$, and is given by $\lambda_{A}=\frac{1}{5}\left(3 c_{\infty}+2 c_{N}\right)$. Beyond $\mathrm{C}$, at lower death rates (eg $\left.\mathrm{B}\right)$, we enter a new phase of growth that includes hypoxia and necrosis. The rapid, transient increase in $R_{H} / R_{N}$ at onset of hypoxia/necrosis (c.f. asymptotics in Appendix A.3) means that for each tumour the width of the proliferating rim decreases until it reaches a steady state and as such $\mathrm{C}$ represents a 'local maximum'. Figure 10 shows the corresponding heatmaps for both the steady state volumes and volume fractions for each constituent of the tumour spheroid.

\section{A.3 Asymptotic Analysis of Model Behaviour Post-radiotherapy}

When a fully developed tumour spheroid including a hypoxic annulus and necrotic core is exposed to fractionated radiation, the composition of the spheroid immediately after irradiation depends on the dose $d$, as described in Sect. 2.2.1. If the dose is sufficiently large, then the post-radiotherapy composition comprises a proliferating rim and a necrotic core, but with the absence of a region of hypoxia. In this scenario, the oxygen concentration in the necrotic core is greater than the threshold for hypoxia (i.e. $\left.c\left(R_{N}\right)>c_{H}\right)$. Upon regrowth, the outer tumour radius, $R$, will increase, while $R_{N}$ will decrease as the necrotic core undergoes decay. Since this parameter regime yields tumour spheroids with proliferating, hypoxic and necrotic compartments prior to radiotherapy, left untreated the tumour will eventually evolve so that the hypoxic annulus will start to redevelop (when $c\left(R_{N}\right)=c_{H}$ ). This sequence of events is summarised in Fig. 11. Simulation results reveal the re-emergence of hypoxia following radiotherapy via a fast, transient increase in $R_{H}$ (see Fig. $4 \mathrm{~b}$ ). Since hypoxic cells are less radio-sensitive, such a rapid increase in the hypoxic volume could have implications for the response to further doses of radiation. We now characterise this behaviour. 


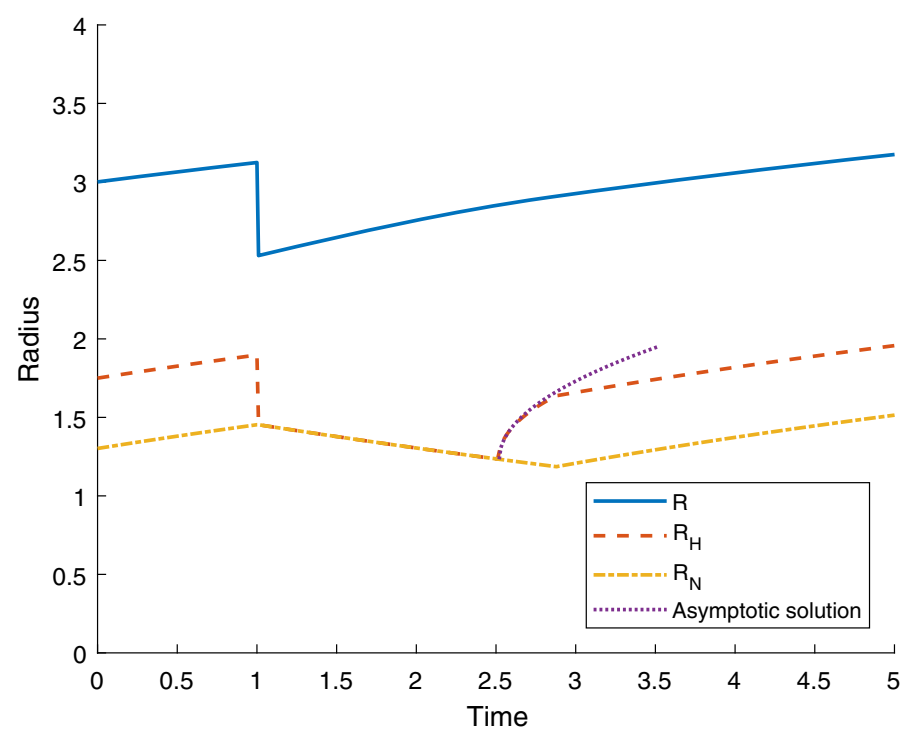

Fig. 12 Asymptotic solution given by Eq. (57) (dotted line) plotted alongside the numerical solution to Eqs. (14)-(23) showing the regrowth of the tumour spheroid after radiotherapy and the fast initial increase in $R_{H}$. We note that during regrowth the mismatch in the oxygen tension on the necrotic core boundary is eventually resolved and the tumour resumes standard Greenspan growth dynamics

The fast initial increase in $R_{H}$ upon regrowth of the tumour spheroid in the Greenspan model after a radiation fraction (see Fig. 12) occurs when the necrotic core is undergoing exponential decay and a region of hypoxia is about to develop outside the necrotic core. We analyse this situation by supposing that (without loss of generality at $t=0) c\left(R_{N}\right)=c_{H}$. Then, solution of Eqs. (14), (18) and (19) yields

$$
c= \begin{cases}c_{\infty}+\frac{\Gamma}{6}\left(R_{N}^{2}-R^{2}\right)+\frac{\Gamma}{3} R_{N}^{3}\left(\frac{1}{R_{N}}-\frac{1}{R}\right), & \text { for } 0<r<R_{N}, \\ c_{\infty}+\frac{\Gamma}{6}\left(r^{2}-R^{2}\right)+\frac{\Gamma}{3} R_{N}^{3}\left(\frac{1}{r}-\frac{1}{R}\right), & \text { for } \quad R_{N}<r<R,\end{cases}
$$

while Eq. (15) reduces to give

$$
\begin{aligned}
\frac{\mathrm{d} R}{\mathrm{~d} t}= & \frac{R}{3}\left[c_{\infty}\left(1-\frac{R_{H}^{3}}{R^{3}}\right)-\left(\lambda_{A}+\lambda_{N} \frac{R_{N}^{3}}{R^{3}}\right)\right] \\
& +\frac{\Gamma}{6} R^{3}\left[\frac{1}{5}\left(1-\frac{R_{H}^{5}}{R^{5}}\right)-\frac{1}{3}\left(1-\frac{R_{H}^{3}}{R^{3}}\right)+2 \frac{R_{N}^{3}}{R^{3}}\left(\frac{1}{6}-\frac{1}{2} \frac{R_{H}^{2}}{R^{2}}+\frac{1}{3} \frac{R_{H}^{3}}{R^{3}}\right)\right],
\end{aligned}
$$

and the internal free boundaries $R_{H}(t)$ and $R_{N}(t)$ satisfy

$$
\left(1-\frac{R_{H}}{R}\right)\left(\frac{R^{2}}{R_{H}^{2}}+\frac{R}{R_{H}}-2 \frac{R_{N}^{3}}{R_{H}^{3}}\right)=\frac{6}{\Gamma R_{H}^{2}}\left(c_{\infty}-c_{H}\right),
$$




$$
R_{N}=R_{N}(0) e^{-\frac{1}{3}\left(\lambda_{A}+\lambda_{N}\right) t}
$$

with $R_{H}(0)=R_{N}(0)$, and $R(0)$ defined by Eq. (45).

With $R_{N}(t)$ defined by Eq. (46), we seek approximate solutions for $R$ and $R_{H}$. Differentiating Eq. (45) with respect to $t$, we obtain an ODE for $R_{H}(t)$, which is singular in the limit as $R_{H} \rightarrow R_{N}$ :

$$
\frac{2}{R_{H}^{2}}\left(R_{H}^{3}-R_{N}^{3}\right) \frac{\mathrm{d} R_{H}}{\mathrm{~d} t}=\left(\frac{R_{H}^{2}}{R^{2}}-2 \frac{R_{N}^{3}}{R^{3}}+2\right) R \frac{\mathrm{d} R}{\mathrm{~d} t}+2\left(\lambda_{A}+\lambda_{N}\right)\left(R-R_{H}\right) \frac{R_{N}^{3}}{R R_{H}} .
$$

We investigate the behaviour in this limit by making the change of variables $S=\frac{R_{H}}{R_{N}}$ with $S=1$ at $t=0$. Then, Eqs. (44) and (47) become

$$
\begin{aligned}
& \frac{\mathrm{d} R}{\mathrm{~d} t}= \frac{R}{3}\left[c_{\infty}\left(1-S^{3} \frac{R_{N}^{3}}{R^{3}}\right)-\left(\lambda_{A}+\lambda_{N} \frac{R_{N}^{3}}{R^{3}}\right)\right]+\frac{\Gamma R^{3}}{6}\left[\frac{1}{5}\left(1-S^{5} \frac{R_{N}^{5}}{R^{5}}\right)-\frac{1}{3}\left(1-S^{3} \frac{R_{N}^{3}}{R^{3}}\right)\right. \\
&\left.+2 \frac{R_{N}^{3}}{R^{3}}\left(\frac{1}{6}-\frac{1}{2} S^{2} \frac{R_{N}^{2}}{R^{2}}+\frac{1}{3} S^{3} \frac{R_{N}^{3}}{R^{3}}\right)\right] \\
&\left(S^{2} \frac{R_{N}^{2}}{R^{2}}-2 \frac{R_{N}^{3}}{R^{3}}+2\right) R \frac{\mathrm{d} R}{\mathrm{~d} t}-2 \frac{R_{N}^{2}}{S^{2}}\left(S^{3}-1\right)\left[\frac{\mathrm{d} S}{\mathrm{~d} t}-\frac{1}{3}\left(\lambda_{A}+\lambda_{N}\right) S\right] \\
& \quad+2\left(\lambda_{A}+\lambda_{N}\right)\left(1-S \frac{R_{N}}{R}\right) \frac{R_{N}^{2}}{S}=0 .
\end{aligned}
$$

We construct approximate solutions to Eqs. (48) and (49) in the limit when $S$ is close to 1 . Introducing the small parameter $\epsilon(0<\epsilon \ll 1)$, we consider the short timescale $t=\epsilon^{2} \tau$ and propose expansions for $S$ and $R$ in this boundary layer of the form

$$
S(\tau) \sim 1+\epsilon S_{1}(\tau)+\epsilon^{2} S_{2}(\tau)+o\left(\epsilon^{3}\right),
$$

and

$$
R(\tau) \sim R_{0}(\tau)+\epsilon R_{1}(\tau)+\epsilon^{2} R_{2}(\tau)+o\left(\epsilon^{3}\right)
$$

The choice of timescale can be justified by a dominant balance argument, allowing us to regularise the ODE for $S$ at leading order. Note that $\frac{\mathrm{d} R}{\mathrm{~d} \tau}=o\left(\epsilon^{2}\right)$, so we deduce that

$$
\begin{aligned}
\frac{\mathrm{d} R_{0}}{\mathrm{~d} \tau}= & 0=\frac{\mathrm{d} R_{1}}{\mathrm{~d} \tau} \\
\frac{\mathrm{d} R_{2}}{\mathrm{~d} \tau}= & \frac{R_{0}}{3}\left[c_{\infty}\left(1-\frac{R_{N 0}^{3}}{R_{0}^{3}}\right)-\left(\lambda_{A}+\lambda_{N} \frac{R_{N 0}^{3}}{R_{0}^{3}}\right)\right]+\frac{\Gamma R_{0}^{3}}{6}\left[\frac{1}{5}\left(1-\frac{R_{N 0}^{5}}{R_{0}^{5}}\right)-\frac{1}{3}\left(1-\frac{R_{N 0}^{3}}{R_{0}^{3}}\right)\right. \\
& \left.+2 \frac{R_{N 0}^{3}}{R_{0}^{3}}\left(\frac{1}{6}-\frac{1}{2} \frac{R_{N 0}^{2}}{R_{0}^{2}}+\frac{1}{3} \frac{R_{N 0}^{3}}{R_{0}^{3}}\right)\right] \\
= & f\left(R_{0}, R_{N 0}\right), \quad \text { say. }
\end{aligned}
$$


Hence, $R \sim R_{0}+\epsilon^{2} R_{2}(\tau)+o\left(\epsilon^{3}\right)$, where $R_{0}=$ const $=R(0)$. We also find that $R_{N}$ has no $o(\epsilon)$ term, since expanding Eq. (46) gives $R_{N} \sim R_{N 0}\left(1-\frac{1}{3}\left(\lambda_{A}+\lambda_{N}\right) \epsilon^{2} \tau+\right.$ $o\left(\epsilon^{4}\right)$ ), where $R_{N 0}=R_{N}(0)$.

Turning our attention back to Eq. (49), we find at leading order

$3 R_{N 0}^{2} S_{1} \frac{d S_{1}}{d \tau}=\left(1-\frac{R_{N 0}^{3}}{R_{0}^{3}}+\frac{1}{2} \frac{R_{N 0}^{2}}{R_{0}^{2}}\right) R_{0} f\left(R_{0}, R_{N 0}\right)+\left(\lambda_{A}+\lambda_{N}\right)\left(1-\frac{R_{N 0}}{R_{0}}\right) R_{N 0}^{2}$

where $f\left(R_{0}, R_{N 0}\right)$ is defined via Eq. (54). Integrating Eq. (55) gives

$$
S_{1}=\left[\frac{1}{3}\left(\left(\frac{1}{R_{0}}-2 \frac{R_{N 0}}{R_{0}^{2}}+2 \frac{R_{0}}{R_{N 0}^{2}}\right) f\left(R_{0}, R_{N 0}\right)+2\left(\lambda_{A}+\lambda_{N}\right)\left(1-\frac{R_{N 0}}{R_{0}}\right)\right)\right]^{\frac{1}{2}} \tau^{\frac{1}{2}} .
$$

So for $t \ll 1$,

$$
\begin{gathered}
R_{H}(t) \sim R_{N 0}+R_{N 0}\left[\frac { 1 } { 3 } \left(\left(\frac{1}{R_{0}}-2 \frac{R_{N 0}}{R_{0}^{2}}+2 \frac{R_{0}}{R_{N 0}^{2}}\right) f\left(R_{0}, R_{N 0}\right)\right.\right. \\
\left.\left.+2\left(\lambda_{A}+\lambda_{N}\right)\left(1-\frac{R_{N 0}}{R_{0}}\right)\right)\right]^{\frac{1}{2}} t^{\frac{1}{2}} .
\end{gathered}
$$

This approximate solution for $R_{H}(t)$ and the numerical solution obtained by solving the full problem are in good agreement (Fig. 12).

As a result of this analysis, we conclude that whenever a hypoxic region re-emerges within a tumour post-radiotherapy, it does so rapidly over a short timescale on which both the outer tumour radius and the radius of the necrotic core do not change dramatically. This phenomenon arises naturally from the model. We note that similar analysis was performed on the original, untreated growth equations by Byrne and Chaplain (1998).

\section{References}

Ahmed KA, Correa CR, Dilling TJ, Rao NG, Shridhar R, Trotti AM, Wilder RB, Caudell JJ (2014) Altered fractionation schedules in radiation treatment: a review. Semin Oncol 41(6):730-750. https://doi.org/ 10.1053/j.seminoncol.2014.09.012. http://linkinghub.elsevier.com/retrieve/pii/S0093775414002322

Alper T, Howard-Flanders P (1956) Role of oxygen in modifying the radiosensitivity of $E$. coli B. Nature 178:978-979

Araujo RP, McElwain DLS (2004) A history of the study of solid tumour growth: the contribution of mathematical modelling. Bull Math Biol 66(5):1039-1091. https://doi.org/10.1016/j.bulm.2003.11. 0023843079723

Breward C, Byrne H, Lewis CE (2003) A multiphase model describing vascular tumour growth. Bull Math Biol 65(4):609-640. https://doi.org/10.1016/S0092-8240(03)00027-2

Byrne HM (2012) Continuum models of avascular tumour growth. In: Antoniouk AV, Melnik RVN (eds) Mathematics and life sciences, chap 12. De Gruyter, pp 279-312

Byrne H, Chaplain M (1998) Necrosis and apoptosis: distinct cell loss mechanisms in a mathematical model of avascular tumour growth. J Theor Med 1:223-235. https://doi.org/10.1080/10273669808833021 
Byrne HM, King JR, McElwain DLS, Preziosi L (2003) A two-phase model of solid tumour growth. Appl Math Lett 16(4):567-573. https://doi.org/10.1016/S0893-9659(03)00038-7

Carlson DJ, Stewart RD, Semenenko VA (2006) Effects of oxygen on intrinsic radiation sensitivity: a test of the relationship between aerobic and hypoxic linear-quadratic (LQ) model parameters. Med phys 33(9):3105-3115. https://doi.org/10.1118/1.2229427

Chvetsov AV, Sandison GA, Schwartz JL, Rengan R (2015) Ill-posed problem and regularization in reconstruction of radiobiological parameters from serial tumor imaging data. Phys Med Biol 60(21):8491-8503. https://doi.org/10.1088/0031-9155/60/21/8491

Enderling H, Chaplain MAJ, Hahnfeldt P (2010) Quantitative modeling of tumor dynamics and radiotherapy. Acta Biotheor 58(4):341-353. https://doi.org/10.1007/s10441-010-9111-z

Eschrich SA, Pramana J, Zhang H, Zhao H, Boulware D, Lee JH, Bloom G, Rocha-Lima C, Kelley S, Calvin DP, Yeatman TJ, Begg AC, Torres-Roca JF (2009) A gene expression model of intrinsic tumor radiosensitivity: prediction of response and prognosis after chemoradiation. Int $\mathrm{J}$ Radiat Oncol Biol Phys 75(2):489-496. https://doi.org/10.1016/j.ijrobp.2009.06.014

Folkman J, Hochberg M (1973) Self-regulation of growth in three dimensions. J Exp Med 138(4):745-753. https://doi.org/10.1084/jem.138.4.745

Fowler JF (2006) Development of radiobiology for oncology—a personal view. Phys Med Biol 51(13):R263-R286. https://doi.org/10.1088/0031-9155/51/13/R16

Frieboes HB, Lowengrub JS, Wise S, Zheng X, Macklin P, Bearer EL, Cristini V (2007) Computer simulation of glioma growth and morphology. NeuroImage 37:S59-S70. https://doi.org/10.1016/j.neuroimage. 2007.03.008. http://linkinghub.elsevier.com/retrieve/pii/S1053811907001784

Gong J, Dos Santos MM, Finlay C, Hillen T (2013) Are more complicated tumour control probability models better? Math Med Biol 30:1-19. https://doi.org/10.1093/imammb/dqr023

Greenspan H (1972) Models for the growth of a solid tumor by diffusion. Stud Appl Math 51:317-340

Grimes DR, Kelly C, Bloch K, Partridge M (2014) A method for estimating the oxygen consumption rate in multicellular tumour spheroids. J R Soc Interface 11(92):20131124. https://doi.org/10.1098/rsif. 2013.1124. http://rsif.royalsocietypublishing.org/content/11/92/20131124.short

Hirschhaeuser F, Menne H, Dittfeld C, West J, Mueller-Klieser W, Kunz-Schughart LA (2010) Multicellular tumor spheroids: an underestimated tool is catching up again. J Biotechnol 148(1):3-15. https://doi. org/10.1016/j.jbiotec.2010.01.012. http://linkinghub.elsevier.com/retrieve/pii/S0168165610000398

Joiner M, van der Kogel A (2009) Basic clinical radiobiology, 4th edn. CRC Press, Boca Raton. https://doi. org/10.1201/b13224

Marcu LG (2010) Altered fractionation in radiotherapy: from radiobiological rationale to therapeutic gain. Cancer Treat Rev 36(8):606-614. https://doi.org/10.1016/j.ctrv.2010.04.004. http://linkinghub. elsevier.com/retrieve/pii/S0305737210000824

McAneney H, O'Rourke SFC (2007) Investigation of various growth mechanisms of solid tumour growth within the linear-quadratic model for radiotherapy. Phys Med Biol 52(4):1039-1054. https://doi.org/ 10.1088/0031-9155/52/4/012

Mueller-Klieser W (1987) Multicellular spheroids_a review on cellular aggregates in cancer research. J Cancer Res Clin Oncol 113(2):101-122. https://doi.org/10.1007/BF00391431

Muriel VP (2006) The impact on oncology of the interaction of radiation therapy and radiobiology. Clin Transl Oncol 8(2):83-93. https://doi.org/10.1007/s12094-006-0163-0

Nilsson P, Thames HD, Joiner MC (1990) A generalized formulation of the 'incomplete-repair' model for cell survival and tissue response to fractionated low dose-rate irradiation. Int J Radiat Biol 57(1):127142. https://doi.org/10.1080/09553009014550401

O'Rourke SFC, McAneney H, Hillen T (2009) Linear quadratic and tumour control probability modelling in external beam radiotherapy. J Math Biol 58(4-5):799-817. https://doi.org/10.1007/s00285-008$0222-y$

Preziosi L, Tosin A (2009) Multiphase modelling of tumour growth and extracellular matrix interaction: mathematical tools and applications. J Math Biol 58(4-5):625-656. https://doi.org/10.1007/s00285008-0218-7

Prokopiou S, Moros EG, Poleszczuk J, Caudell J, Torres-Roca JF, Latifi K, Lee JK, Myerson R, Harrison LB, Enderling H (2015) A proliferation saturation index to predict radiation response and personalize radiotherapy fractionation. Radiat Oncol 10(1):159. https://doi.org/10.1186/s13014-015-0465-x. http://www.ro-journal.com/content/10/1/159 
Rockne RC, Frankel P (2017) Mathematical modeling in radiation oncology. In: Wong JYC, Schultheiss TE, Radany EH (eds) Advances in radiation oncology, Springer International Publishing, chap 12, pp 255-271

Sachs RK, Hahnfeld P, Brenner DJ (1997) The link between low-LET dose-response relations and the underlying kinetics of damage production/repair/misrepair. Int J Radiat Bioly 72(4):351-374. https:// doi.org/10.1080/095530097143149

Sachs RK, Hlatky LR, Hahnfeldt P (2001) Simple ODE models of tumor growth and anti-angiogenic or radiation treatment. Math Comput Model 33(12-13):1297-1305. https://doi.org/10.1016/S08957177(00)00316-2

Schaller G, Meyer-Hermann M (2006) Continuum versus discrete model: a comparison for multicellular tumour spheroids. Philos Trans R Soc A Math Phys Eng Sci 364(1843):1443-1464. https://doi.org/ 10.1098/rsta.2006.1780. http://rsta.royalsocietypublishing.org/cgi/doi/10.1098/rsta.2006.1780

Sutherland R, Carlsson J, Durand R, Yuhas J (1981) Spheroids in cancer research. Cancer Res 41(July):2980_ 2984. http://cancerres.aacrjournals.org/content/41/7/2980.abstract

Thrall DE (1997) Biologic basis of radiation therapy. Vet Clin N Am Small Anim Pract 27(1):21-35

Torre LA, Bray F, Siegel RL, Ferlay J, Lortet-tieulent J, Jemal A (2015) Global cancer statistics, 2012. CA Cancer J Clin 65(2):87-108. https://doi.org/10.3322/caac.21262

Withers HR (1999) Radiation biology and treatment options in radiation oncology. Cancer Res 59(7 Suppl):1676s-1684s 